\title{
THREE DIMENSIONAL MECHANICAL MODEL FOR SIMULATING THE NSM FRP STRIPS SHEAR STRENGTH CONTRIBUTION TO RC BEAMS
}

\author{
Vincenzo Bianco ${ }^{1}$, J.A.O. Barros ${ }^{2}$ and Giorgio Monti ${ }^{3}$
}

\begin{abstract}
Shear strengthening of Reinforced Concrete (RC) beams by means of Near Surface Mounted (NSM) Fiber Reinforced Polymer (FRP) strips is an emerging technique for structural rehabilitation that is gaining increasing interest in the FRP community mainly because of some advantages it provides with respect to the better consolidated technique of the Externally Bonded Reinforcement (EBR). Those advantages encompass, mainly, a better exploitation of material and a higher protection against vandalism along with a relative faster applicability. Yet, the behavior of such NSM FRP strips is extremely complex, as can be gathered by experimental evidence, due to the complex geometry, the non linear mechanical properties of bond, and the scatter affecting the concrete tensile properties along with their non-linearity. In an attempt to provide valuable contribution to a better understanding of their behavior, a three dimensional mechanical model for simulating the shear strength contribution provided by a system of NSM FRPs to a RC beam throughout the loading process was recently developed. Its upgraded version is herein presented along with the main findings. It correctly interprets the experimental evidence taking into account complex phenomena such as the interaction between bond transferred force and concrete fracture along with the interaction between adjacent strips.
\end{abstract}

Keywords: Numerical Model; FRP; NSM; Shear Strengthening; Concrete Fracture; Debonding; Tensile Rupture.

\section{Introduction}

Shear strengthening of RC beams by NSM technique consists of gluing FRP strips by a powerful structural adhesive into thin shallow slits cut onto the concrete cover of the web lateral faces. The most recent experimental works (Dias and Barros 2008, De Lorenzis and Rizzo 2006) devoted at appraising the effectiveness of such technique spotlighted the occurrence of a peculiar failure mode consisting of the progressive detachment and outward expulsion of the concrete cover from the underlying beam core. That failure mode was even more

\footnotetext{
${ }^{1}$ PhD Student, Dept. of Structural Engrg. and Geotechnics, Sapienza University of Rome, via A. Gramsci 53, 00197 Rome, Italy. E-mail: vincenzo.bianco@uniroma1.it.

${ }^{2}$ Associate Professor, Dept. of Civil Engineering, University of Minho, Campus de Azurém, 4810-058 Guimarães, Portugal. E-mail: barros@civil.uminho.pt, corresponding Author. Tel. +351 253510 210, Fax. +351 253510217

${ }^{3}$ Full Professor, Dept. of Structural Engrg. and Geotechnics, Sapienza University of Rome, via A. Gramsci 53, 00197 Rome, Italy. E-mail: giorgio.monti@uniroma1.it.
} 
pronounced in the case of low strength concrete beams (Dias et al. 2007). A subsequent experimental-analytical investigation (Bianco et al. 2007a) demonstrated, even by means of an analogy with adhesive anchors, due to the relatively still short amount of experimental results regarding NSM FRP strips, that such failure mode can be ascribed to the semi conical tensile fracture of concrete surrounding each NSM strip (Fig. 1). When principal tensile stresses transferred to the surrounding concrete exceed its tensile strength, concrete fractures along a surface, envelope of the compression isostatics, whose shape can be conveniently assumed as semi conical. Totally, depending on the relative mechanical and geometrical properties of the materials involved, the possible failure modes affecting the behavior, at ultimate, of NSM strips comprise: debonding, tensile rupture of the strip itself, concrete semi conical tensile fracture and a mixed shallow-semi-cone-plus-debonding failure mode (Fig. 1). The term debonding is adopted to designate loss of bond that is failure occurring within the adhesive layer or just a few millimeters inside the surrounding concrete so as the strip pull out results (Fig. 1). During the loading process of a RC beam subject to shear, when the concrete average tensile strength $f_{c t m}$ is overcome at the web intrados, some shear cracks originate therein and successively progress towards the web extrados. Those cracks can be thought of as a single Critical Diagonal Crack (CDC) inclined of an angle $\theta$ with respect to the beam longitudinal axis (Fig. 2). The CDC can be schematized as an inclined plane dividing the web into two portions sewn together by the crossing strips (Fig. 2a). At load step $t_{1}$, the two web parts separated by the CDC start moving apart by pivoting around the crack end (point E in Fig. 2b). From that step on, by increasing the applied load, the CDC opening angle $\gamma\left(t_{n}\right)$ progressively widens. The strips crossing the CDC oppose its widening by anchoring to the surrounding concrete to which they transfer, by bond, the force originating at their intersection with the CDC, $O_{i}^{l}$, and due to the imposed end slip $\delta_{L i}\left[\gamma\left(t_{n}\right)\right]$. The capacity of each strip is provided by its available bond length $L_{f i}$ that is the shorter between the two parts into which the crack divides its actual length $L_{f}$. As the spacing between subsequent strips $s_{f}$ is reduced, their semi-conical fracture surfaces overlap and the resulting envelope area progressively becomes smaller than the mere summation of each of them (see Fig. 3a). This detrimental interaction between strips can be easily taken into account by calculating the resulting semi-conical surface ascribed to each strip accordingly. For very short values of the spacing, the resulting concrete failure surface is almost parallel to the web face of the beam, which is in agreement with the failure mode observed experimentally, consisting in the detachment of the concrete cover from the underlying beam core (see Fig. 13). Since the position of those semi-conical surfaces is symmetric with respect to the vertical plane passing through the beam axis, the horizontal outward components of the tensile strength vectors 
distributed throughout their surfaces are balanced only from an overall standpoint but not locally (Fig. 3b). This local unbalance of the horizontal tensile stress component orthogonal to the beam web face justifies the outward expulsion of the concrete cover in both the uppermost and lowermost parts of the strengthened sides of the web (Fig. 13).

\section{Main algorithm}

The algorithm adopted (Fig. 4), takes as input both geometrical and mechanical parameters i.e.: depth $h_{w}$ and width $b_{w}$ of the strengthened beam web; inclination of both the Critical Diagonal Crack (CDC) angle $\theta$ and the strips $\beta$ with respect to the beam longitudinal axis; strips' spacing measured along the beam axis $s_{f}$; angle $\alpha$ between axis and generatrices of the semi-conical fracture surface; concrete average compressive strength $f_{c m}$; strips' tensile strength $f_{f u}$ and Young's modulus $E_{f}$; thickness $a_{f}$ and width $b_{f}$ of the strip's cross section; values of bond stress $\tau_{0}, \tau_{1}, \tau_{2}$ and slip $\delta_{1}, \delta_{2}, \delta_{3}$ defining the adopted local bond stress-slip relationship; increment $\dot{\gamma}$ and maximum value $\gamma_{\max }$ of the opening angle of the CDC. The algorithm, taken as input the information above, is meant to determine, for each $k$-th possible geometrical configurations that the occurred CDC could assume with respect to the FRPs, the NSM shear strength contribution as function of the crack opening angle $V_{f, k}\left[\gamma\left(t_{n}\right)\right]$ throughout the loading process $\left(t_{n}: t_{1} \rightarrow t_{s}\right)$. Geometrical information in the global reference system oxyz (Fig. 2a) regarding each $k$-th configuration is stored in the matrix $\underline{x}$ that has dimension $k \times 2$. The first column stores the value $x_{f 1, k}$ that is the position of the first strip, for the $k$-th configuration, measured along the $x$ axis of $o x y z$ and the second column stores the integer number of strips $\left(N_{f, k}\right)$ crossing the CDC for the $k$-th configuration. The number (real) of strips that can intersect the CDC, is $N_{f, \text { real }}=h_{w} \cdot(\cot \theta+\cot \beta) / s_{f}$. The number (integer) of strips that can actually cross the CDC can assume two values: $N_{f \text {,int }}^{l}$ and $N_{f \text {,int }}^{h}$, differing by one unit and determinable respectively by rounding off $N_{f \text {,real }}$ to the lowest and to the highest integer. In the case in which $N_{f \text {,real }}$ is an integer, $N_{f \text {,int }}^{h}=N_{f, \text { real }}$ and $N_{f \text {,int }}^{l}=N_{f, \text { real }}-1$. The above numbers $N_{f \text {,int }}^{l}$ and $N_{f \text {,int }}^{h}$ result to be, in an order not definable a priori and function of the values assumed by $h_{w}, \theta$, and $s_{f}$, an odd and an even number or vice-versa, herein indicated as $N_{f, \text { odd }}$ and $N_{f, e v}$. Even if the possible values of the number of strips that can effectively intersect the crack are

two only, it is necessary to maintain both the above denominations $\left(N_{f \text {,int }}^{l}\right.$ and $N_{f \text {,int }}^{h}$ or $N_{f, \text { odd }}$ and $\left.N_{f, e v}\right)$ in 
order to single out more easily the possible configurations assumed by the strips with respect to the crack. In particular, in the present work, three geometrical configurations are examined $(k=1,2,3)$, defined as follows: ( $k=1$ ) the minimum number of strips ( $N_{f \text {,int }}^{l}$ ) with the first one located at a distance equal to the spacing from the crack origin $\left(x_{f 1,1}=s_{f}\right)$; $(k=2)$ an even number of strips $\left(N_{f, e v}\right)$ symmetrically placed with respect to the crack axis $\left(x_{f 1,2}=0.5 \cdot\left[L_{f} \cdot \sin (\theta+\beta) / \sin \theta-\left(N_{f, e v}-1\right) \cdot s_{f}\right]\right) ;(k=3)$ an odd number of strips $\left(N_{f, \text { odd }}\right)$ with the central one attaining the maximum length by being located along the crack axis $\left(x_{f 1,3}=0.5 \cdot\left[h_{w} \cdot(\cot \theta+\cot \beta)-\left(N_{f, o d d}-1\right) \cdot s_{f}\right]\right)$. The matrix $\underline{F}_{k}$ is also determined for each $k$-th configuration and storing in the first column the position of the $i$-th strip:

$x_{f i, k}=x_{f 1, k}+(i-1) \cdot s_{f}$

and in the second the corresponding value of the available bond length:

$L_{f, k}= \begin{cases}{\left[x_{f 1, k}+(i-1) \cdot s_{f}\right] \cdot \frac{\sin \theta}{\sin (\theta+\beta)}} & \text { for } x_{f i, k}<\frac{h_{w}}{2} \cdot(\cot \theta+\cot \beta) \\ L_{f}-\left[x_{f 1, k}+(i-1) \cdot s_{f}\right] \cdot \frac{\sin \theta}{\sin (\theta+\beta)} & \text { for } x_{f i, k} \geq \frac{h_{w}}{2} \cdot(\cot \theta+\cot \beta)\end{cases}$

After having determined the geometry in $o x y z$, calculations are executed for each $k$. The following four matrices are built:

- $\underline{H}_{k}$ that is a $N_{f, k} \times 2$ dimensions matrix whose first column stores the position of the $i$-th strip with respect to the assumed crack origin $H_{i 1}=x_{f i, k}$. The second column stores the relevant concrete semi-cone height $L_{f i, k}^{c}\left(t_{n} ; q_{m}\right)$ that is the portion of $L_{f i, k}$ around which the surrounding concrete has fractured along a semi conical surface, as further specified hereafter and that is initialized with zero: $L_{f i}^{c}=0.0$;

- $\underline{L}_{R}$ that is a $N_{f, k} \times 1$ vector storing, in the $i$-th cell, the relevant resisting bond length $L_{R f i, k}\left(t_{n} ; q_{m}\right)$ that is the portion of $L_{f i, k}$ still bonded to concrete initialized with the available bond length i.e. $L_{R f i, k}=L_{f i, k}$;

- $\underline{u}$ that is a $N_{f, k} \times 1$ vector storing, in the $i$-th cell, information regarding the state of the relevant strip that is if it has already reached its ultimate state $\left(u_{i}=1\right)$ or not $\left(u_{i}=0\right)$. The initial assumption is made that they do not reach their ultimate state;

- $\underline{F}_{k}$ that is a $N_{f, k} \times t_{s}$ matrix storing the height of the semi-conical fracture surface occurred, if any, for each strip of the $k$-th configuration.

After having determined and stored that initial information, for each load step $t_{n}$, the following vector is built: 
- $\underline{\delta}_{L}\left(t_{n}\right)$ that is a $N_{f, k} \times 1$ vector storing, in each $i$-th row, the value of the imposed end slip applied to the relevant $i$-th strip parallel to its orientation $\delta_{L i}$. In the present work it is assumed a linear trend of imposed slip along the CDC length (see Fig. 2b).

Within each step ( $t_{n}: t_{1} \rightarrow t_{s}$ ), for each $i$-th strip $\left(i: 1 \rightarrow N_{f, k}\right.$ ), the Strip Function determines the current values of the strength of the strip parallel to its orientation $V_{f i, k}^{p}\left[\gamma\left(t_{n}\right)\right]$, and the up-dated value of the parameters providing information regarding the state of the strip i.e.: $u_{i, k}\left(t_{n+1}\right), L_{R f i, k}\left(t_{n+1}\right)$ and $L_{f i, k}^{c}\left(t_{n+1}\right)$. The matrices $\underline{H}_{k}, \underline{L}_{R, k}$ and $\underline{u}_{k}$ are then updated and the NSM shear strength contribution is incremented:

$V_{f, k}\left[\gamma\left(t_{n}\right)\right] \leftarrow V_{f, k}\left[\gamma\left(t_{n}\right)\right]+2 \cdot V_{f i, k}^{p} \cdot \sin \beta$

The finial output of the main algorithm are the three vectors $\underline{V}_{f, k}$ and the one storing the values of the CDC opening angle $\underline{\gamma}$.

\section{Single strip contribution}

Within the Strip Function (Figs. 5-6), an iterative procedure $\left(q_{m}: q_{1} \rightarrow q_{e}\right)$ is carried out in order to determine the equilibrium condition $\left(q_{e}\right)$ in the concrete surrounding the $i$-th strip depending on the imposed end slip $\left(\delta_{L i}\left(t_{n}\right)\right)$, its current state $\left(u_{i}\left(t_{n}\right), L_{R f i}\left(t_{n}\right)\right)$ and the current state of concrete fracture regarding all of the strips $\left(\underline{H}_{k}\left(t_{n}\right)\right)$. That iteration is performed as long as neither the surrounding concrete has reached equilibrium $\left(c_{e}=0\right)$ nor the strip has ruptured $\left(u_{i}=0\right)$. Within each iteration $\left(q_{m}\right)$ the current value of the transfer length ( $\left.L_{t r, i}\left[\delta_{L i}\left(t_{n}\right) ; L_{R f i}\left(t_{n} ; q_{m}\right)\right]\right)$ is evaluated and scanned to check if the progressive value of the force transferred by bond $\left(V_{f i}^{b d}\left[\delta_{L i}\left(t_{n}\right) ; L_{R f i}\left(t_{n} ; q_{m}\right) ; x_{i}^{t r}\right]\right)$ is such as to exceed the corresponding value of the concrete fracture capacity

$\left(V_{f i}^{c f}\right)$. The transfer length is evaluated and discretized in $n_{d}\left(n_{n}: n_{1} \rightarrow n_{d}\right)$ segments $\left(\underline{x}_{i}^{t r}\left(n_{d} \times 1\right)\right)$ by the Bond Function that also provides a vector storing the corresponding progressive values of the bond transferred force $\left(\underline{V}_{f i}^{b d}\left(n_{d} \times 1\right)\right)$. The progressive value of $V_{f i}^{c f}$, evaluated in correspondence of the incremental value of the $i$-th strip available bond length $\left(X_{i}^{l}=L_{f i}^{c}+x_{i}^{t r}\left(n_{n}\right)\right)$, is evaluated within the function Concrete Fracture Capacity by spreading $f_{c t m}$ (calculable from $f_{c m}$ by literature relationships) throughout the semi-conical surface with height $X_{i}^{l}$, orthogonally to it in each point and integrating according to the following formula (Fig. 6c): 


$$
V_{f i}^{p, c f}=\int_{C_{f i}\left(X_{i}^{l} ; \alpha\right)}\left(f_{c t m} \cdot \sin \alpha\right) \cdot d C_{f i}
$$

where $C_{f i}$ concisely denotes the semi-conical surface. Eq. (4) can be reduced to the evaluation of the area of the semi-ellipse ( $\left.E_{f i}\right)$ intersection of the semi-cone with the crack plane as follows (Bianco et al. 2006):

$$
V_{f i}^{p, c f}=\sin (\theta+\beta) \cdot f_{c t m} \cdot \int_{E_{f i}\left(X_{i}^{l} ; \alpha\right)} d E_{f i}
$$

In general, due to the interaction among adjacent strips, $V_{f i}^{p, c f}$ also depends on the length of the semi-cones that have already formed along each $j$-th $\left(j: 1 \rightarrow N_{f, k}\right)$ of all the strips as further specified below but, for the simplified case in which the $i$-th strip is not affected by the interaction with other strips and it results orthogonal to the crack plane (Fig. 6c) Eq. (5) simplifies into: $V_{f i}^{c f}=0.5 \cdot \pi \cdot f_{c t m} \cdot \operatorname{tg}^{2} \alpha \cdot\left(X_{i}^{l}\right)^{2}$. After having scanned the transfer length and having taken note of the eventual occurrence of concrete fracture $\left(c_{f} \leftarrow 1\right)$ and the height of the occurred semi-conical fracture ( $H \leftarrow X_{i}^{l}$ ), one of the following alternatives might be the case:

- Concrete has fractured $\left(c_{f}=1\right)$ but the fracture has not reached the inner tip $\left(H<L_{f i}\right)$. In that case, it is taken note of the up-dated length of the cone $\left(L_{f i}^{c} \leftarrow H\right.$ ), of the resisting bond length $\left(L_{R f i} \leftarrow\left(L_{f i}-H\right)\right.$ ) and iteration is performed $\left(q_{m} \leftarrow q_{m+1}\right)$;

- Concrete has fractured $\left(c_{f}=1\right)$ and the fracture has reached the inner tip $\left(H=L_{f i}\right)$. Note is taken of the updated value of both semi-cone length and resisting bond length, as in the case above, and of the $i$-th strip having reached its ultimate state $\left(u_{i, k} \leftarrow 1\right)$. The $i$-th strip shear strength contribution is $V_{f i}^{p}\left(t_{n}\right)=V_{f i}^{c f}\left(L_{f i}\right)$;

- Concrete has not fractured $\left(c_{f}=0\right)$ and the actual value of bond transferred force is lower than the strip tensile rupture capacity $\left(V_{f i}^{b d}\left(n_{d}\right)<V_{f}^{t r}\right)$. In this case equilibrium in concrete is reached $\left(c_{e}=1\right)$ and there is no need to iterate and the strip strength can be returned $V_{f i}^{p}\left(t_{n}\right)=V_{f i}^{b d}\left(n_{d}\right)$;

- Concrete has not fractured $\left(c_{f}=0\right)$ and the actual value of bond transferred force is higher or equal to the strip tensile rupture capacity $\left(V_{f i}^{b d}\left(n_{d}\right) \geq V_{f}^{t r}\right.$ ). In this case equilibrium in concrete is reached $\left(c_{e}=1\right)$ and there is no need to iterate, the strip strength can be returned $\left(V_{f i}^{p}\left(t_{n}\right)=V_{f}^{t r}\right)$ and note can be taken of the strip's reaching its ultimate state $\left(u_{i, k} \leftarrow 1\right)$. 


\section{Progressive bond transferred force}

The Bond Function (Fig. 7) has to return two vectors $\underline{x}_{i}^{t r}$ and $\underline{V}_{f i}^{b d}$, both of dimensions $n_{d} \times 1$, storing the progressive values $x_{i}^{t r}\left(n_{n}\right)$ of the discretized transfer length $L_{t r, i}\left(L_{R f i} ; \delta_{L i}\right)$ and the corresponding values of the progressive force transferred to the surrounding concrete by bond $V_{f i}^{b d}\left[L_{R f i} ; \delta_{L i} ; x_{i}^{t r}\left(n_{n}\right)\right]$, respectively. It was recently demonstrated (Bianco et. al 2007c, 2008b), also by means of comparison between experimental recordings and analytical predictions, that the employment of bond and the successive and progressive loss of bond (debonding), by imposing an increasing end slip to an NSM FRP strip, can be thought of as a "constant wave” i.e. a constant distribution of slip and tangential stress progressing from the loaded end inwards, towards the free extremity (Fig. 8). From an analytical standpoint, for the given value of $\delta_{L i}$, the governing differential equation is first solved for an infinite value of the resisting bond length determining the trend of slip $\delta(x)$, tangential stress $\tau(x)$, axial stress in the strip $\sigma_{f}(x)$ and axial stress along the corresponding transfer length $L_{t r}\left(\delta_{L i}\right)$. After that, the actual value of the transfer length $L_{t r, i}\left(L_{R f i} ; \delta_{L i}\right)$ is determined, and then discretization and integration are performed. The governing differential equation is the following (Bianco et al. 2007c):

$$
\frac{d^{2} \delta}{d x^{2}}-\tau[\delta(x)] \cdot J_{1}=0 \text { with } J_{1}=\frac{L_{p}}{A_{f}} \cdot\left(\frac{1}{E_{f}}+\frac{A_{f}}{A_{c} \cdot E_{c}}\right)
$$

where $L_{p}\left(2 \cdot b_{f}+a_{f}\right)$ is the effective bond perimeter of the strip cross-section, $A_{f}\left(a_{f} \cdot b_{f}\right)$ is the strip cross section area and $A_{c}\left(s_{f} \cdot b_{w} \cdot 0.5\right)$ is the area of the cross section of the concrete prism, amount of the surrounding concrete on which the strip is glued and attributed to the $i$-th strip. Eq. (6) is obtained by taking into consideration: a) the equilibrium relationships $\frac{d \sigma_{f}(x)}{d x}-\tau(x) \cdot \frac{L_{p}}{A_{f}}=0$ and $\sigma_{f}(x) \cdot A_{f}+\sigma_{c}(x) \cdot A_{c}=0$; b) the constitutive equations for the adhesive layer $\tau=\tau(\delta)$ and the two adhering materials $\sigma_{f}=E_{f} \cdot \frac{d u_{f}}{d x}$ and $\sigma_{c}=E_{c} \cdot \frac{d u_{c}}{d x} ;$ c) kinematic compatibility $\delta(x)=u_{f}(x)-u_{c}(x)$ where $u_{f}(x)$ and $u_{c}(x)$ are the punctual displacements of the strip and concrete, respectively. The relationship $\tau=\tau(\delta)$ herein adopted (Bianco et. al 2007c, 2008b) is the following (Fig. 9): 


$$
\tau(\delta)=\left\{\begin{array}{lc}
\tau_{0}+\frac{\tau_{1}-\tau_{0}}{\delta_{1}} \cdot \delta & 0 \leq \delta \leq \delta_{1} \\
\tau_{1}-\frac{\tau_{1}-\tau_{2}}{\delta_{2}-\delta_{1}} \cdot\left(\delta-\delta_{1}\right) & \delta_{1}<\delta \leq \delta_{2} \\
\tau_{2}-\frac{\tau_{2}}{\delta_{3}-\delta_{2}} \cdot\left(\delta-\delta_{2}\right) & \delta_{2}<\delta \leq \delta_{3} \\
0 & \delta>\delta_{3}
\end{array}\right.
$$

Once the relationship $\delta(x)$ has been obtained by solving Eq. (6) with the convenient boundary conditions, the expressions for the stress in the strip and the tangential stress along this latter can be deduced as follows:

$\sigma_{f}(x)=J_{2} \cdot \frac{d \delta}{d x}$ with $J_{2}=\frac{E_{f} \cdot E_{c} \cdot A_{c}}{E_{c} \cdot A_{c}+E_{f} \cdot A_{f}}$

and:

$\tau(x)=J_{3} \cdot \frac{d^{2} \delta}{d x^{2}}$ with $J_{3}=\frac{E_{f} \cdot A_{f} \cdot E_{c} \cdot A_{c}}{L_{p} \cdot\left(E_{c} \cdot A_{c}+E_{f} \cdot A_{f}\right)}$

\section{Solution of Bond for an infinite resisting bond length}

\section{Elastic phase}

When the imposed end slip is $\delta_{L i} \leq \delta_{1}$, the expression of the interfacial slip, obtained by solving Eq. (6) written in the local reference system $o x^{e}$ originating in the leftward unloaded extremity of the transfer length (Fig. 8) and imposing the boundary conditions $\delta^{e}=0$ at $x^{e}=0$ and $\delta^{e}=\delta_{L i}$ at $L_{t r}^{e}\left(\delta_{L i}\right)$, is the following:

$\delta^{e}\left(x^{e}\right)=C_{1}^{e} \cdot e^{\lambda \cdot x^{e}}+C_{2}^{e} \cdot e^{-\lambda \cdot x^{e}}-\frac{\tau_{0} \cdot J_{1}}{\lambda^{2}}$

with $C_{1}^{e}=\left[\delta_{L i}+\frac{\tau_{0} \cdot J_{1}}{\lambda^{2}} \cdot\left(1-e^{-\lambda \cdot L_{t r}^{e}}\right)\right] \cdot \frac{1}{e^{\lambda \cdot L_{t r}^{e}}-e^{-\lambda \cdot L_{t r}^{e}}}, C_{2}^{e}=\frac{\tau_{0} \cdot J_{1}}{\lambda^{2}}-C_{1}^{e}$ and $\frac{1}{\lambda^{2}}=\frac{\delta_{1}}{\left(\tau_{1}-\tau_{0}\right) \cdot J_{1}} \cdot \quad$ By $\quad$ imposing $\quad$ the equilibrium condition $L_{p} \cdot \int_{0}^{L_{t r}^{e}} \tau^{e}\left(x^{e}\right) \cdot d x^{e}=A_{f} \cdot \sigma_{f}\left(L_{t r}^{e}\right)$, the expression of the transfer length for the first phase can be obtained as function of the imposed slip:

$L_{t r}\left(\delta_{L i}\right)=L_{t r}^{e}\left(\delta_{L i}\right)=\frac{1}{\lambda} \cdot \operatorname{arcosh} \frac{B^{e}}{2 \cdot A^{e}}$

with $A^{e}=\frac{\tau_{0} \cdot J_{1}}{2 \cdot \lambda^{2}}$ and $B^{e}=\delta_{L i}+\frac{\tau_{0} \cdot J_{1}}{\lambda^{2}}$. The transfer length at the end of the elastic phase $L_{t r 1}$ and the corresponding value of force transferred to concrete $V_{1}^{b d}$, both invariants for given input parameters, are obtained imposing $\delta_{L i}=\delta_{1}$ : 
$L_{t r 1}=L_{t r}^{e}\left(\delta_{1}\right) ; V_{1}^{b d}=V^{b d, e}\left(\delta_{1}\right)=L_{p} \cdot \int_{0}^{L_{t r 1}} \tau^{e}\left(x^{e}\right) \cdot d x^{e}$

\section{Softening Phase}

When the imposed end slip is $\delta_{1}<\delta_{L i} \leq \delta_{2}$, the expression for the interfacial slip, obtained by solving Eq. (6) written in the reference system $o x^{s}$ originating at the point of the bond length where slip is equal to $\delta_{1}$ (Fig. 8), with boundary conditions $\delta^{s}=\delta_{1}$ at $x^{s}=0$ and $\delta^{s}=\delta_{L i}$ at $x^{s}=L_{t r}^{s}$, is the following:

$\delta^{s}\left(x^{s}\right)=C_{1}^{s} \cdot \sin \left(\beta \cdot x^{s}\right)+C_{2}^{s} \cdot \cos \left(\beta \cdot x^{s}\right)+\frac{\tau_{1} \cdot J_{1}}{\beta^{2}}+\delta_{1}$

with $C_{1}^{s}=\frac{1}{\sin \left(\beta \cdot L_{t r}^{s}\right)} \cdot\left\{\delta_{L i}-\delta_{1}+\frac{\tau_{1} \cdot J_{1}}{\beta^{2}} \cdot\left[\cos \left(\beta \cdot L_{t r}^{s}\right)-1\right]\right\}, C_{2}^{s}=-\frac{\tau_{1} \cdot J_{1}}{\beta^{2}}$ and $\frac{1}{\beta^{2}}=\frac{\left(\delta_{2}-\delta_{1}\right)}{\left(\tau_{1}-\tau_{2}\right) \cdot J_{1}}$. The expression of the transfer length $L_{t r}^{s}\left(\delta_{L i}\right)$ corresponding to the amount of the infinite bond length undergoing softening is:

$L_{t r}^{s}\left(\delta_{L i}\right)=\frac{1}{\beta} \cdot \phi+\frac{1}{\beta} \cdot \arcsin \frac{C^{s}}{\left[\left(A^{s}\right)^{2}+\left(B^{s}\right)^{2}\right]^{1 / 2}}$

with $A^{s}=V_{1}^{b d}, B^{s}=J_{3} \cdot L_{p} \cdot \frac{\tau_{1} \cdot J_{1}}{\beta}, C^{s}=J_{3} \cdot L_{p} \cdot \beta \cdot\left(\delta_{L i}-\delta_{1}-\frac{\tau_{1} \cdot J_{1}}{\beta^{2}}\right)$ and $\phi=\arcsin \frac{B^{s}}{\left[\left(A^{s}\right)^{2}+\left(B^{s}\right)^{2}\right]^{1 / 2}}$. The overall transfer length, for $\delta_{1}<\delta_{L i} \leq \delta_{2}$, is then:

$L_{t r}\left(\delta_{L i}\right)=L_{t r 1}+L_{t r}^{s}\left(\delta_{L i}\right)$

The maximum value of the transfer length that can undergo softening and the relevant value of the force transferred to the surrounding concrete are the following invariants:

$L_{t r 2}=L_{t r}^{s}\left(\delta_{2}\right) ; V_{2}^{b d}=V^{b d, s}\left(\delta_{2}\right)=L_{p} \cdot \int_{0}^{L_{t r 2}} \tau^{s}\left(x^{s}\right) \cdot d x^{s}$

\section{Softening Friction Phase}

When the imposed slip is larger than the value at which softening friction begins, $\delta_{2}<\delta_{L i} \leq \delta_{3}$, the expression for the interfacial slip, obtained by solving Eq. (5) written for a reference system $o x^{s f}$ originating at the point of the infinite bond length where slip is equal to $\delta_{2}$ (Fig. 8) and with boundary conditions $\delta^{s f}=\delta_{2}$ at $x^{s f}=0$ and $\delta^{s f}=\delta_{L i}$ at $x^{s f}=L_{t r}^{s f}$ is:

$\delta^{s f}\left(x^{s f}\right)=C_{1}^{s f} \cdot \sin \left(\gamma \cdot x^{s f}\right)+C_{2}^{s f} \cdot \cos \left(\gamma \cdot x^{s f}\right)+\delta_{3}$ 
with $C_{1}^{s f}=\frac{1}{\sin \left(\gamma \cdot L_{t r}^{s f}\right)} \cdot\left[\delta_{L i}-\delta_{3}+\left(\delta_{3}-\delta_{2}\right) \cdot \cos \left(\gamma \cdot L_{t r}^{s f}\right)\right], C_{2}^{s f}=\delta_{2}-\delta_{3}$ and $\frac{1}{\gamma^{2}}=\frac{\left(\delta_{3}-\delta_{2}\right)}{\tau_{2} \cdot J_{1}}$. The expression of the transfer length $L_{t r}^{s f}\left(\delta_{L i}\right)$ corresponding to the amount of length undergoing softening friction is:

$L_{t r}^{s f}\left(\delta_{L i}\right)=\frac{1}{\gamma} \cdot \psi+\frac{1}{\gamma} \cdot \arcsin \frac{C^{s f} \cdot}{\left[\left(A^{s f}\right)^{2}+\left(B^{s f}\right)^{2}\right]^{1 / 2}}$

with $A^{s f}=V_{1}^{b d}+V_{2}^{b d}, B^{s f}=J_{3} \cdot L_{p} \cdot \gamma \cdot\left(\delta_{3}-\delta_{2}\right), C^{s f}=J_{3} \cdot L_{p} \cdot \gamma \cdot\left(\delta_{L i}-\delta_{3}\right)$ and $\quad \psi=\arcsin \frac{B^{s f}}{\left[\left(A^{s f}\right)^{2}+\left(B^{s f}\right)^{2}\right]^{1 / 2}}$.

The overall transfer length, for $\delta_{2}<\delta_{L i} \leq \delta_{3}$, is:

$L_{t r}\left(\delta_{L i}\right)=L_{t r 1}+L_{t r 2}+L_{t r}^{s f}\left(\delta_{L i}\right)$

The maximum value of the infinite bond length that can undergo softening friction and the relevant value of the force transferred to the surrounding concrete are:

$$
L_{t r 3}=L_{t r}^{s f}\left(\delta_{3}\right) ; V_{3}^{b d}=V^{b d, s f}\left(\delta_{3}\right)=L_{p} \cdot \int_{0}^{L_{t r 3}} \tau^{s f}\left(x^{s f}\right) \cdot d x^{s f}
$$

\section{Free Slipping Phase}

When the imposed slip is larger than the value at which free slipping begins, $\delta_{L i}>\delta_{3}$, the expression for the interfacial slip, obtained by solving Eq. (5) written for a reference system $o x^{f_{s}}$ originating at the point of the bond length where slip is equal to $\delta_{3}$ (Fig. 8) and with boundary conditions $\delta^{f s}=\delta_{3}$ at $x^{f s}=0$ and $\delta^{f s}=\delta_{L i}$ at $x^{f s}=L_{t r}^{f s}$ is:

$\delta^{f s}\left(x^{f s}\right)=C_{1}^{f s} \cdot x^{f s}+C_{2}^{f s}$

with $C_{1}^{f s}=\frac{\delta_{L i}-\delta_{3}}{L_{t r}^{f s}}$ and $C_{2}^{f s}=\delta_{3}$. The expression of the transfer length $L_{t r}^{f s}\left(\delta_{L i}\right)$ corresponding to the amount of length undergoing free slipping is:

$L_{t r}^{f s}\left(\delta_{L i}\right)=J_{3} \cdot L_{p} \cdot \frac{\delta_{L i}-\delta_{3}}{V_{1}^{b d}+V_{2}^{b d}+V_{3}^{b d}}$

The overall transfer length, for $\delta_{L i}>\delta_{3}$, and the force transferred by bond to the surrounding concrete, are:

$L_{t r}\left(\delta_{L i}\right)=\sum_{p=1}^{3} L_{t r p}+L_{t r}^{f s}\left(\delta_{L i}\right) ; V^{b d}\left(\delta_{L i}\right)=\sum_{p=1}^{3} V_{p}^{b d}$

\section{Solution of Bond for the actual value of the resisting bond length}


After having solved the governing differential equation for the actual value of the imposed slip $\delta_{L i}$ and having thereby determined the relevant transfer length $L_{t r}\left(\delta_{L i}\right)$ and trend of bond stress $\tau(x)$ for the case of an infinite resisting bond length, the actual value of the transfer length $L_{t r, i}\left(L_{R f i} ; \delta_{L i}\right)$ is determined as the minimum between $L_{R f i}\left(t_{n}\right)$ and $L_{t r}\left(\delta_{L i}\right)$. The transfer length is then discretized and the progressive value of force transferred by bond is determined by integrating the tangential stress according to the following general formulation:

$V_{f i}^{b d}\left[x_{i}^{t r}\left(n_{n}\right)\right]=L_{p} \cdot\left[\int \tau^{s f}\left(x^{s f}\right) \cdot d x^{s f}+\int \tau^{s}\left(x^{s}\right) \cdot d x^{s}+\int \tau^{e}\left(x^{e}\right) \cdot d x^{e}\right]$

where the integration extremities are omitted since they depend on both the phase undergone by the free end and the current value of the progressive abscissa $x_{i}^{t r}\left(n_{n}\right)$ along the transfer length (Figs. 7-8). For instance, Eq. (24) assumes, for the case $b$ represented in Fig. 8, the following explicit form: $V_{f i}^{b d}\left(x_{i}^{t r}\right)=L_{p} \cdot\left[\int_{L_{t r}\left(\delta_{L i}\right)-\left(L_{t r 1}+x_{i}^{t r}\right)}^{L_{t r 2}} \tau^{s}\left(x^{s}\right) \cdot d x^{s}+\int_{0}^{x_{2}^{s f}} \tau^{s f}\left(x^{s f}\right) \cdot d x^{s f}\right]$ with $x_{2}^{s f}<x_{i}^{t r} \leq\left(x_{2}^{s f}+L_{t r 2}\right)$ and $x_{2}^{s f}=L_{t r}\left(\delta_{L i}\right)-\left(L_{t r 1}+L_{t r 2}\right)$.

\section{Concrete Fracture Capacity}

In the most general case in which the $i$-th strip progressive concrete fracture capacity $V_{f i}^{c f}\left[X_{i}^{l}\left(n_{n}\right)\right]$ is influenced by the semi-conical fracture surfaces that have already occurred around all of the strips up to that moment ( $L_{f j}^{c}\left(t_{n} ; q_{m}\right) \forall j=1, ., N_{f k}$ and $j \neq i$ ) (Fig. 6d) its evaluation becomes more complex. Anyway, the simplification adopted in Eq. (5) that reduces the evaluation of the semi-conical surface area to the area of the semi-ellipse, intersection of the semi-conical surface with the CDC plane, results extremely powerful to correctly quantify interaction among strips. In the most general case, (Fig. 11 that represents the situation depicted in Fig. 6d), that interaction can be either mono-directional, longitudinal or transversal, or bi-directional. The longitudinal interaction can occur when, due to the reduced spacing with respect to the height of the web, the semi-cones associated to adjacent strips located at the same side of the web, and consequently their relevant semi-ellipses, overlap along their major semi-axis (see for instance the semi-ellipses 1 and 3 of the example of Fig. 11). The transversal interaction can occur when, for slender beam cross sections of high $h_{w} / b_{w}$ ratio, the semi-ellipses symmetrically placed on the opposite sides of the web, intersect each other along their minor semi-axis (see the semi-ellipse 2 of Fig. 11). In this latter case, the area of the $i$-th semi-ellipse is limited, upwards, by the line $Y=b_{w} / 2$, i.e. the trace, on the CDC plane (with reference system $O X Y$ ), of the vertical plane passing through the beam axis. In case bidirectional interaction occurred, the area on the CDC plane associated to the $i$-th strip, 
would be composed of two terms: $\mathcal{A}_{f i}^{\text {nlin }}$, limited upwards by the non-linear branch of the relevant semi-ellipse $Y_{i}(X)$ and another, $\mathcal{A}_{f i}^{l i n}$, limited by the line $Y=b_{w} / 2$. Hence, due to the bi-directional interaction, the area of the semi-ellipse associated to the $i$-th strip is calculated as follows:

$$
\int_{E_{f i}\left(X_{i}^{l} ; \alpha_{f i}\right)} d E_{f i}=\left(\mathcal{A}_{f i}^{n l i n}+\mathcal{A}_{f i}^{l i n}\right)
$$

Within the function labeled Concrete Fracture Capacity, the two areas $\mathcal{A}_{f i}^{\text {lin }}$ and $\mathcal{A}_{f i}^{\text {nlin }}$ are evaluated by a geometrical closed-form algorithm (Bianco et al. 2006) that briefly consists of: (1) writing the equation of each $j$ th semi-ellipse associated to the $j$-th strip's latest semi-conical fracture surface ( $j: 1 \rightarrow N_{f, k}$ ), if it has formed, in the CDC reference system, (2) evaluating and storing in some auxiliary vectors the abscissa of the points that might constitute integration extremities for the $i$-th semi-ellipse, (3) suitably selecting the integration extremities for both the linear and non-linear integration range of the $i$-th semi-ellipse and (4) integrating, as hereafter specified (Fig. 11). For the sake of brevity, all of the analytical details are herein omitted but they can be found elsewhere (Bianco et al. 2006).

\section{Definition of the geometric quantities in $O X Y Z$}

To easily determine the equations of the semi-ellipses in $O X Y$, the prominent geometrical quantities, for each $j$ th strip $\left(j=1, ., N_{f, k}\right)$, are evaluated and stored in the corresponding $j$-th row of the $\underline{G}$ matrix, of dimensions $N_{f, k} \times 8$. The cells relative to those strips for which concrete fracture has not occurred yet, are filled with a "non-value" that can be, for instance, an asterisk that has no physical meaning at all. Note in fact, that a "non-value” term cannot be zero, for instance, since this latter has a physical meaning representing the position, in $O X Z$, of the assumed crack origin. The columns from the first to the eighth of $\underline{G}$ store, respectively (see Fig. 11a): the position $X_{f j}$ of each strip singled out along the $O X$ axis of the crack plane reference system $O X Y$; the length $a_{j}$ of the major semi-axis of the resulting semi-ellipse; the value $X_{o j}$ of the position, along the $O X$ axis, of the center of the $j$-th semi-ellipse; the fourth and fifth columns store, respectively, the value of the abscissa $e_{1 P j}$ and of the ordinate $e_{2 P j}$, in the local reference system of the $j$-th semi-ellipse $o e_{1 j} e_{2 j} e_{3 j}$, of an auxiliary point $P$ necessary to write the equation of the relevant ellipse; the sixth column stores the value of the length $b_{j}$ of the minor semi-axis of the semi-ellipse; the seventh and eighth columns store respectively the values of the position, along the $O X$ axis, of the leftward $v_{1 j}$ and of the rightward $v_{2 j}$ vertices of the semi-ellipse along its major axis. 


\section{Determination of the coefficients of the semi-ellipses}

The equation of a generic $j$-th semi-ellipse $Y_{j}(X)$ in the crack plane reference system has to be determined:

$Y_{j}(X)=+\sqrt{-\frac{\left(E_{j 1} \cdot X^{2}+E_{j 3} \cdot X+E_{j 4}\right)}{E_{j 2}}}$

For this purpose, the coefficients $E_{j 1}, E_{j 2}, E_{j 3}, E_{j 4}$ of the $j$-th semi-ellipses are evaluated and stored in the $j$-th row of the $\underline{E}$ matrix that has $N_{f, k} \times 4$ dimensions.

\section{Determination of the auxiliary vectors of integration points}

It is worth determining, even if they are not strictly necessary for the implementation of the algorithm, some auxiliary vectors i.e. $\underline{X}^{p 1}, \underline{X}^{p 2}, \underline{X}^{q}, \underline{Y}^{e}, \underline{M}, \underline{N}, \underline{Q}$ since they condense some operations that, otherwise, should be repeated several times. $\underline{X}^{p 1}$ and $\underline{X}^{p 2}$ are two $1 \times N_{f, k}$ dimension vectors containing, respectively, the abscissa of the first, $X_{i j}^{p 1}$, and second, $X_{i j}^{p 2}$, intersection points, if actually existing, between the $i$-th and each other $j$-th semi-ellipse. For the sake of generality, the case in which the two semi-ellipses should intersect each other in two points is envisaged. The cell corresponding to a non-existent intersection is filled with a "nonvalue”. $\underline{X}^{q}$ is a $1 \times 2$ dimensions vector containing the abscissa of the left $X_{i 1}^{q}$ and right $X_{i 2}^{q}$ intersection, if actually existing, of the $i$-th semi-ellipse with the straight line $Y=b_{w} / 2 . \underline{Y}^{e}$ is a $1 \times 2$ dimensions vector containing, the ordinate values $Y_{i 1}^{e}$ and $Y_{i 2}^{e}$ assumed by the $i$-th semi-ellipse in correspondence of $X=0$ and $X=L_{d}$, respectively, if the semi-ellipse actually passes through those abscissa values.

$\underline{M}, \underline{N}, \underline{Q}$ are $1 \times N_{f, k}$ dimension matrices containing, respectively, the coefficients $M_{i j}, N_{i j}$ and $Q_{i j}$ with $j=1, ., N_{f, k}$, calculated as follows:

$M_{l j}=\left[\left(\frac{E_{l 1}}{E_{l 2}}-\frac{E_{j 1}}{E_{j 2}}\right)\right] ; N_{l j}=\left[\left(\frac{E_{l 3}}{E_{l 2}}-\frac{E_{j 3}}{E_{j 2}}\right)\right] ; Q_{l j}=\left[\left(\frac{E_{l 4}}{E_{l 2}}-\frac{E_{j 4}}{E_{j 2}}\right)\right]$

where $E_{i 1}, E_{i 2}, E_{i 3}, E_{i 4}$ and $E_{j 1}, E_{j 2}, E_{j 3}, E_{j 4}$ are, respectively, the coefficients of the $i$-th and $j$-th semi-ellipses stored in the relevant rows of the $\underline{E}$ matrix.

Determination of the integration points in the non linear range $\underline{X}^{\text {nlin }}$

$\underline{X}^{n l i n}$ is a $1 \times n^{\text {nlin }}$ dimensions vector containing the couples of abscissa values constituting limits of the integration intervals for the $i$-th semi-ellipse equation $Y_{i}(X)$ where $n^{\text {nlin }}$ is the maximum number of real values 
of integration limits for the $i$-th semi-ellipse (an even number). To evaluate $\underline{X}^{\text {nlin }}$, five other auxiliary vectors $\underline{X}^{\text {nlin } 1}, \underline{X}^{\text {nlin2 }}, \underline{X}^{\text {nlin3 }}, \underline{X}^{\text {nlin4 }}, \underline{X}^{\text {nlin5 }}$ have to be determined, based on both the auxiliary ones $\underline{X}^{p 1}, \underline{X}^{p 2}, \underline{X}^{q}, \underline{Y}^{e}, \underline{M}, \underline{N}, \underline{Q}$, output of the previous block of calculations, and the matrix of the semi-ellipses geometrical properties, $\underline{G}$.

$\underline{X}^{n l i n 1}$ and $\underline{X}^{n l i n 2}$ are two $1 \times N_{f k}$ dimensions vectors containing the abscissa values $X_{i j}^{n l i n 1}$ and $X_{i j}^{n l i n 2}$, amongst those already calculated and stored, respectively, in the auxiliary vectors $\underline{X}^{p 1}$ and $\underline{X}^{p 2}$, that effectively constitute useful integration limits for the $i$-th semi-ellipse equation. The explicit analytical details of the acceptance condition can be found elsewhere (Bianco et al. 2006). Cells corresponding to a discarded point are filled with a “non-value”.

$\underline{X}^{n \operatorname{lin} 3}$ is a $1 \times 2$ vector containing, in the first and second cell, $X_{i 1}^{n \operatorname{lin} 3}$ and $X_{i 2}^{n \operatorname{lin} 3}$, the abscissa values of the left and right intersection points of the $i$-th semi-ellipse with the straight line $Y=b_{w} / 2$ that result effective for the integration of the corresponding equation $Y_{i}(X)$.

$\underline{X}^{n l i n 4}$ is a $1 \times 2$ dimensions vector containing, in the first cell, the null abscissa value, $X_{i 1}^{\text {nlin } 4}=0$, and the crack length $L_{d}$ in the second cell, $X_{i 2}^{\text {nlin4 }}=L_{d}$, if those values result to be effective integration limits for the relevant semi-ellipse $Y_{i}(X)$.

$\underline{X}^{n l i n 5}$ is a $1 \times 2$ dimensions vector containing the abscissa of the vertices of the major semi-axis of the $i$-th semi-ellipse that constitute effective integration extremities for the $i$-th ellipse. The first and second cell term, $X_{i 1}^{n \operatorname{lin} 5}$ and $X_{i 2}^{n \operatorname{lin} 5}$ of the $\underline{X}^{\text {nlin5 }}$ vector have to be set equal to the term $G_{i 7}$ and $G_{i 8}$, respectively, stored in the seventh column cell of the corresponding $i$-th row of the matrix $\underline{G}$ if they satisfy the acceptance conditions.

The number of columns of the $\underline{X}^{\text {nlin }}$ vector, $n^{\text {nlin }}$, is equal to the maximum number of real abscissa values constituting effective integration limits for the $i$-th semi-ellipse equation, i.e.:

$n^{\text {nlin }}=$ realnumbers $\left\{X_{i}^{n l i n 1} ; X_{i}^{n l i n 2} ; X_{i}^{n l i n 3} ; X_{i}^{n l i n 4} ; X_{i}^{n l i n 5}\right\}$

The $\underline{X}^{\text {nlin }}$ matrix is then built by joining the effective terms, discarding the "non-values", present in the auxiliary vectors $\underline{X}^{n l i n 1}, \underline{X}^{n l i n 2}, \underline{X}^{n l i n 3}, \underline{X}^{n l i n 4}, \underline{X}^{n l i n 5}$ and sorting them in increasing order. For instance, the vector $\underline{X}^{n l i n}$ for the second strip of Fig. 11 is $\underline{X}^{n l i n}=(1 \times 4)=\left[\begin{array}{llll}0.0 & X_{21}^{q} & X_{22}^{q} & X_{23}^{p 1}\end{array}\right]$. 


\section{Determination of the integration points in the linear range $\underline{X}^{\text {lin }}$}

$\underline{X}^{\text {lin }}$ is a $1 \times n^{\text {lin }}$ dimensions vector containing the couples of abscissa values constituting limits of the integration intervals, in correspondence of the $i$-th semi-ellipse, of the equation $Y=b_{w} / 2$ where $n^{\text {lin }}$ is the maximum number of real values of integration limits for the $i$-th semi-ellipse (an even number). To evaluate $\underline{X}^{\text {lin }}$, four other auxiliary matrices $\underline{X}^{\operatorname{lin} 1}, \underline{X}^{\operatorname{lin} 2}, \underline{X}^{\operatorname{lin} 3}, \underline{X}^{\operatorname{lin} 4}$ have to be determined, based on the auxiliary ones $\underline{X}^{p 1}$, $\underline{X}^{p 2}, \underline{X}^{q}, \underline{Y}^{e}, \underline{M}, \underline{N}, \underline{Q}$, already built.

$\underline{X}^{l i n 1}$ and $\underline{X}^{\operatorname{lin} 2}$ are two $1 \times N_{f, k}$ dimensions vectors containing, the abscissa values, amongst those already calculated and stored in the auxiliary vectors $\underline{X}^{p 1}$ and $\underline{X}^{p 2}$, respectively, that effectively constitute useful integration limits for the linear range ascribed to the $i$-th semi-ellipse. The general $j$-th cell $X_{i j}^{l i n 1 / 2}$ of the $\underline{X}^{l i n 1 / 2}$ vector is set equal to the corresponding term $X_{i j}^{p 1 / 2}$ of the corresponding auxiliary vector $\underline{X}^{p 1 / 2}$ if $X_{i j}^{p 1 / 2}$ is such as to satisfy, for the $i$-th semi-ellipse, the acceptance conditions (Bianco et al. 2006). Note that $X_{i j}^{p 1 / 2}$ represents the two possible solutions, $X_{i j}^{p 1}$ and $X_{i j}^{p 2}$.

$\underline{X}^{l i n 3}$ is a $1 \times 2$ dimensions vector containing, in the first and second columns, $X_{i 1}^{l i n 3}$ and $X_{i 2}^{l i n 3}$, respectively, the abscissa values of the left $X_{i 1}^{q}$ and right $X_{i 2}^{q}$ intersection points of the $i$-th semi-ellipse with the straight line $Y=b_{w} / 2$ that result effective for the integration of the corresponding equation $Y=b_{w} / 2$.

$\underline{X}^{l i n 4}$ is a $1 \times 2$ dimensions vector containing, in the first cell, the null abscissa value, $X_{i 1}^{l i n 4}=0$, and the $L_{d}$ value in the second cell, $X_{i 2}^{\text {lin } 4}=L_{d}$, if those values result to be effective integration limits for the linear range ascribed to the $i$-th semi-ellipse.

The number of columns of the $\underline{X}^{\text {lin }}$ matrix, $n^{\text {lin }}$, is equal to the maximum number of real abscissa values constituting effective integration limits for the corresponding $i$-th semi-ellipse in the linear range, i.e.:

$n^{l i n}=$ realnumbers $\left\{X_{i}^{\operatorname{lin} 1} ; X_{i}^{\operatorname{lin} 2} ; X_{i}^{\operatorname{lin} 3} ; X_{i}^{l i n 4}\right\}$

The $\underline{X}^{\text {lin }}$ matrix is then built by joining the effective terms, discarding the "non-values" present in the auxiliary vectors $\underline{X}^{\operatorname{lin} 1}, \underline{X}^{\operatorname{lin} 2}, \underline{X}^{\operatorname{lin} 3}, \underline{X}^{\operatorname{lin} 4}$, and sorting them in increasing order. For instance, the vector $\underline{X}^{\text {lin }}$ for the second strip of Fig. 11 becomes $\underline{X}^{l i n}=\left[\begin{array}{ll}X_{21}^{q} & X_{22}^{q}\end{array}\right]$.

\section{Determination of the area and strength}


The non-linear area $\mathcal{A}_{f i}^{\text {nlin }}$ ascribed to the $i$-th semi-ellipse, is equal to:

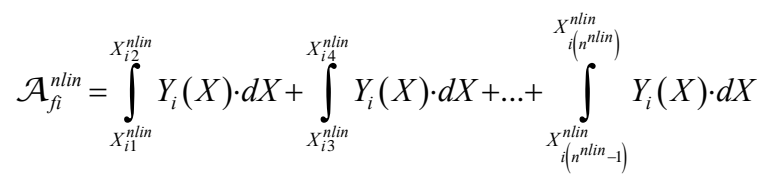

For the sake of brevity, the expression of the exact integration of the equation of the semi-ellipse is omitted but it can be found elsewhere (Bianco et al. 2006). The term $\mathcal{A}_{f i}^{\text {lin }}$ can be obtained from:

$$
\mathcal{A}_{f i}^{l i n}=\int_{X_{i 1}^{l i n}}^{X_{i 2}^{l i n}} \frac{b_{w}}{2} \cdot d X+\int_{X_{i 3}^{l i n}}^{X_{i 4}^{l i n}} \frac{b_{w}}{2} \cdot d X+\ldots . . \int_{\left.X_{i\left(n^{l i n}\right.}^{l i n}-1\right)}^{X_{i\left(n^{l i n}\right)}^{l i n}} \frac{b_{w}}{2} \cdot d X
$$

Note that in the above Eqs. (30) and (31) the abscissa values, already stored in $\underline{X}^{\text {nlin }}$ and $\underline{X}^{\text {lin }}$, respectively, have to be considered integration limits by pairs in sequence.

\section{Model Appraisal}

The proposed model was applied to the RC beams tested by Dias et al. (2007) and by Dias and Barros (2008). The beams tested in those experimental programs were $\mathrm{T}$ cross-section RC beams characterized by the same test set-up, the same amount of longitudinal reinforcement, the same kind of CFRP strips and epoxy adhesive and they differed for the mechanical properties of concrete. In fact, the former experimental program was characterized by a concrete mean tensile strength $f_{c m}$ of $18.6 \mathrm{MPa}$ while the latter by $31.1 \mathrm{MPa}$. Both series presented different configurations of NSM strips, in terms of both inclination $\beta$ and spacing $s_{f}$ and the former program also included beams characterized by a different amount of existing steel stirrups. The details of the beams taken as basis of the appraisal are listed in Table 1. Those beams are characterized by the following common geometrical and mechanical parameters: $b_{w}=180 \mathrm{~mm} ; h_{w}=300 \mathrm{~mm} ; \quad f_{f u}=2952 \mathrm{MPa}$; $E_{f}=166 \mathrm{GPa} ; a_{f}=1.4 \mathrm{~mm} ; b_{f}=10.0 \mathrm{~mm}$. The parameters characterizing the adopted local bond stress-slip relationship, being the average values of those obtained in a previous investigation (Bianco et al. 2007c and 2008b) are: $\tau_{0}=2.0 \mathrm{MPa} ; \tau_{1}=20.1 \mathrm{MPa} ; \tau_{2}=9.0 \mathrm{MPa} ; \delta_{1}=0.07 \mathrm{~mm} ; \delta_{2}=0.83 \mathrm{~mm} ; \delta_{3}=14.1 \mathrm{~mm}$. The CDC inclination angle $\theta$ adopted in the simulations plotted in Fig. 12 is the one experimentally observed and reported in Table 1 . The angle $\alpha$ was assumed equal to $28.5^{\circ}$, being the average of values obtained in a previous investigation (Bianco et al. 2006) by back analysis of experimental data. The two parameters characterizing the loading process are: $\dot{\gamma}=0.01^{\circ}$ and $\gamma_{\max }=1.0^{\circ}$. Concrete average tensile strength $f_{c t m}$ was calculated from the 
average compressive strength by means of the formulae present in the CEB Fib Model Code 1990 resulting in 1.45 $\mathrm{MPa}$ and 2.45 $\mathrm{MPa}$ for the former and latter series of beams, respectively.

Comparison between the numerical results and experimental recordings, for the beams listed in Table 1 , are plotted in Fig. 12. From that comparison, a satisfactory data-fitting performance of the proposed model, in terms of prediction of the NSM shear strength contribution $V_{f, k}$ arises, regardless of the different concrete mechanical properties, inclination of the strips, their spacing along the beam axis and amount of existing stirrups.

It has to be outlined that the difference between the peak value of $V_{f}$, obtained for the three different configurations, and consequently the dispersion of the numerical predictions with respect to the experimental recording, increases by reducing the spacing between adjacent strips (see Fig. 12).

The typical graph of shear strength contribution as function of the CDC opening angle $V_{f}\left[\gamma\left(t_{n}\right)\right]$ is characterized by abrupt decays which correspond to the failure of the strips. The peculiar behavior of a RC beam strengthened in shear by NSM technique can be easily explained referring to one of those beams as for instance the beam labeled 2S-7LI45-II whose cracking scenario, both numerically predicted and experimentally recorded is reported in Fig. 13. The first strips to fail are those characterized by shorter available bond lengths that generally fail in the first stages of the loading process, like for instance: the $1^{\text {st }}\left(\gamma=0.02^{\circ}\right)$ and the $5^{\text {th }}$ $\left(\gamma=0.03^{\circ}\right)$ of the $1^{\text {st }}$ configuration (Fig. 13a); the $1^{\text {st }}$ and $6^{\text {th }}\left(\gamma=0.01^{\circ}\right)$ and the $2^{\text {nd }}\left(\gamma=0.02^{\circ}\right)$ of the $2^{\text {nd }}$ configuration (Fig. 13b). Those failures are not so evident in the corresponding graph (Fig. 12) since, in the first load steps, the contribution provided by the strips with a higher available bond length is increasing and relatively much higher. When a strip fails at a higher stage of the loading process, the corresponding decay in the load carrying capacity, is much more evident, like happens, for instance: for the $2^{\text {nd }}$ strip of the $1^{\text {st }}$ configuration at $\gamma=0.07^{\circ}$, the $3^{\text {rd }}$ of the $2^{\text {nd }}$ configuration at $\gamma=0.07^{\circ}$ or the $3^{\text {rd }}$ strip of the $3^{\text {rd }}$ configuration at $\gamma=0.19^{\circ}$.

The first two ones are mixed shallow-semi-cone-plus-debonding failures and the third is characterized by a semiconical concrete fracture that reaches the inner tip. After those failures, the corresponding graphs, present a different trend: in the first two cases, a maximum relative follows meanwhile, in the third, the shear carrying capacity goes on diminishing in a smooth way. The former behavior is due to the fact that, when the last fracture occurs, that is the mixed failure of the $2^{\text {nd }}$ and $3^{\text {rd }}$ strip respectively, the remaining strips still have a resisting bond length higher than the required transfer length and their contribution can still increase before gradual complete debonding follows. The latter is due to the fact that, when the $3^{\text {rd }}$ central strip fails, the $2^{\text {nd }}$ and the $4^{\text {th }}$, 
had already failed by mixed failure so as, the overall carrying capacity goes on diminishing up to the complete debonding of their left resisting bond lengths.

The numerical modeling strategy herein proposed also lets parametric studies be carried out in order to assess the influence of all of the involved parameters on the NSM shear strength contribution. Herein, for the sake of brevity, only a small parametric study is presented (Fig. 14a-b) that aims at singling out, even by means of the comparison between numerical predictions and experimental recordings, the influence of the spacing for beams with strips at $60^{\circ}$ and with two different kinds of concrete. It arises that, as expected, the higher the concrete mechanical properties, the higher the shear carrying capacity, for the same value of spacing between adjacent strips. It can also be gathered that, by reducing the spacing between adjacent strips, due to the increase of the number of strips effectively crossing the CDC, the shear strength contribution increases even if, as highlighted in

Fig. 14c for the $3^{\text {rd }}$ configuration only (with $\beta=60^{\circ} ; f_{c m}=31.1 M P a$ ) the smaller the spacing, the higher the group effect. This latter can be defined as the decrease of shear strength contribution with respect to an ideal situation in which, the same system of strips, characterized by the real value of the spacing $s_{f}$, the same available bond lengths and the same imposed end slips, are spaced out, along the CDC, at such an extent that they do not interact any longer between each other. The corresponding increase in shear strength contribution increases up to a maximum ideal value beyond which, any further increase of the ideal spacing between adjacent strips does not produce any further increase in carrying capacity. That can be also gathered from Fig. 13d in which the ideal trend is plotted as function of the ideal spacing for the real configuration of strips at $s_{f}=75 \mathrm{~mm}$.

The detrimental group effect increases by reducing the spacing between strips (Fig. 14c).

\section{Conclusions}

The need to provide a rational explanation to the observed peculiar failure mode affecting the behavior, at ultimate, of RC beam strengthened in shear by the NSM technique led to the development of a comprehensive numerical model for simulating the NSM shear strength contribution throughout the loading process. The upgraded version of that model was herein presented. The comparison between the numerical predictions and the experimental recordings showed the high level of accuracy of the proposed model especially if it is taken into consideration that: the model neglects the softening behavior of concrete in tension, the high scatter affecting concrete tensile strength and, on the contrary, the simplified and indirect way in which it was herein calculated.

The application of that model also let some complex phenomena such as the group effect between adjacent strips to be pinpointed. Despite its relative complexity, the proposed model can be usefully applied to single out 
interesting information for designers interested in applying such front-line technique. At the same time, it can be conveniently summarized into a simplified closed-form design formula for practitioners.

\section{Acknowledgements}

The authors of the present work wish to acknowledge the support provided by the "Empreiteiros Casais”, S\&P®, degussa ${ }^{\circledR}$ Portugal, and Secil (Unibetão, Braga). The study reported in this paper forms a part of the research program "SmartReinforcement - Carbon fibre laminates for the strengthening and monitoring of reinforced concrete structures” supported by ADI-IDEIA, Project n 13-05-04-FDR-00031. Also, this work was carried out under the auspices of the Italian DPC-ReLuis Project (repertory n. 540), Research Line 8, whose financial support is greatly appreciated. 


\section{References}

Bianco, V., Barros, J.A.O., Monti, G., (2006). “Shear Strengthening of RC beams by means of NSM laminates: experimental evidence and predictive models”, Technical report 06-DEC/E-18, Dep. Civil Eng., School Eng. University of Minho, Guimarães- Portugal.

Bianco, V., Barros, J.A.O., Monti, G., (2007a). “A new approach for modeling the NSM shear strengthening contribution in reinforced concrete beams”, FRPRCS-8, University of Patras, Greece,16-18 July, ID 8-12.

Bianco, V., Barros, J.A.O., Monti, G., (2007b). “Influence of the concrete mechanical properties on the efficacy of the shear strengthening intervention on RC beams by NSM technique”, Asia-Pacific Conference on FRP in Structures, University of Hong Kong, China,12-14 December.

Bianco, V., Barros, J.A.O., Monti, G., (2007c). "Shear Strengthening of RC beams by means of NSM strips: a proposal for modeling debonding”, Technical report 07-DEC/E-29, Dep. Civil Eng., School Eng. University of Minho, Guimarães- Portugal.

Bianco, V., Barros, J.A.O., Monti, G., (2008a). “Analytical modeling strategy for predicting the NSM FRP strips contribution to RC beams shear strength”, Challenges for Civil Constructions, CCC 2008, Faculty of Engineering of the University of Porto, Porto, Portugal, 16-18 April.

Bianco, V., Barros, J.A.O., Monti, G., (2008b). “A proposal for modeling debonding of NSM FRP strips for shear Strengthening of RC beams”, $6^{\text {th }}$ International Conference AMCM 2008, Analytical Models and new Concepts in Concrete and Masonry Structures, Lodz, 9-11 June.

De Lorenzis, L., Rizzo, A., (2006). “Behaviour and capacity of RC beams strengthened in shear with NSM FRP reinforcement”, 2nd Int. fib Congress, Naples-Italy, June 5-8, Paper ID 10-9 in CD.

Dias, S.J.E., Bianco, V., Barros, J.A.O., Monti, G., (2007). "Low strength concrete T cross section RC beams strengthened in shear by NSM technique”, Workshop-Materiali ed Approcci Innovativi per il Progetto in Zona Sismica e la Mitigazione della Vulnerabilità delle Strutture, University of Salerno, Italy, 12-13 February.

Dias, S.J.E. and Barros, J.A.O., (2008). "Shear Strengthening of T Cross Section Reinforced Concrete Beams by Near Surface Mounted Technique”, Journal of Composites for Construction, ASCE, Vol. 12, No. 3, pp. 300-311.

Sena-Cruz, J.M. (2004). “Strengthening of concrete structures with near-surface mounted CFRP laminate strips” PhD Thesis, Department of Civil Engineering, University of Minho, Guimarães-Portugal. 
Sena-Cruz, J.M., Barros, J.A.O., (2004). “Bond between near-surface mounted CFRP laminate strips and concrete in structural strengthening”, Journal of Composites for Construction, ASCE, Vol. 8, No. 6, pp. 519-527. 


\section{TABLE CAPTIONS}

Table 1. Values of the parameters characterizing beams adopted to appraise the proposed model.

Table 1. Values of the parameters characterizing beams adopted to appraise the proposed model.

\begin{tabular}{|c|c|c|c|c|c|c|c|c|c|c|}
\hline $\begin{array}{l}\text { Beam } \\
\text { Label }\end{array}$ & Series & $\begin{array}{c}\theta^{\exp } \\
\circ\end{array}$ & $\begin{array}{c}\beta \\
\circ\end{array}$ & $\begin{array}{c}s_{f} \\
m m\end{array}$ & $\begin{array}{l}f_{c m} \\
M P a\end{array}$ & $\begin{array}{c}\text { Steel } \\
\text { Stirrups }\end{array}$ & $\begin{array}{c}V_{f, 1}^{\max } \\
k N\end{array}$ & $\begin{array}{c}V_{f, 1}^{\max } \\
k N\end{array}$ & $\begin{array}{c}V_{f, 1}^{\max } \\
k N\end{array}$ & $\begin{array}{c}V_{f}^{\exp } \\
k N\end{array}$ \\
\hline 2S-5LV-I & $\begin{array}{l}\text { Dias \& } \\
\text { Barros } \\
(2006)\end{array}$ & 40 & 90 & 160 & 31.1 & $\phi 6 / 300 \mathrm{~mm}$ & 52.33 & 26.42 & 55.34 & 25.20 \\
\hline 2S-8LV-I & “ & 36 & “ & 100 & “ & “ & 68.58 & 58.88 & 64.33 & 48.60 \\
\hline 2S-3LI60-I & “ & 33 & 60 & 325 & “ & “ & 50.69 & 18.90 & 51.68 & 35.40 \\
\hline 2S-7LI60-I & “ & 33 & “ & 139 & “ & “ & 52.98 & 63.07 & 67.58 & 54.60 \\
\hline 2S-4LI45-II & $\begin{array}{c}\text { Dias et al. } \\
\text { (2007) }\end{array}$ & 40 & 45 & 275 & 18.6 & $\phi 6 / 300 \mathrm{~mm}$ & 25.06 & 21.89 & 37.30 & 33.90 \\
\hline 2S-7LI45-II & “ & 30 & “ & 157 & “ & “ & 49.36 & 47.13 & 45.95 & 48.00 \\
\hline 4S-4LI45-II & “ & 40 & “ & 275 & “ & $\phi 6 / 180 \mathrm{~mm}$ & 25.06 & 21.89 & 37.3 & 26.04 \\
\hline 4S-7LI45-II & “ & 40 & “ & 157 & “ & “ & 40.58 & 37.48 & 40.63 & 31.56 \\
\hline
\end{tabular}




\section{FIGURE CAPTIONS}

Fig. 1. Possible failure modes of an NSM FRP strip: (a) debonding, (b) laminate tensile rupture, (c) concrete semi-conical fracture, (d) mixed shallow semi-cone plus debonding.

Fig. 2. RC beam web: a) axonometric view of the adopted schematization and b) shear loading process.

Fig. 3. Interaction among adjacent strips: a) axonometric view and b) section parallel to the CDC plane.

Fig. 4. Main algorithm: flow chart.

Fig. 5. Strip Function: flow chart.

Fig. 6. Strip Function: (a-b) iterative procedure for searching the equilibrium condition in the surrounding concrete; (c) evaluation of the progressive concrete fracture capacity for a single slip in a simplified case and (d) in presence of interaction between adjacent strips not orthogonal to the CDC plane.

Fig. 7. Flow chart of the Bond Function.

Fig. 8. Invariant bond wave: (a) evaluated for an infinite bond length; progressing from the loaded end to the free extremity for successive values of the imposed end slip $\delta_{L i}\left(t_{n}\right)$ (b1) and $\delta_{L i}\left(t_{n+1}\right)$ (b2).

Fig. 9. Assumed local bond stress-slip relationship.

Fig. 10. Concrete Fracture Capacity Function: flow chart.

Fig. 11. CDC plane: (a) geometrical quantities in $O X Y$ and the ellipse local reference system $o_{j} e_{1 j} e_{2 j}$; (b) abscissa values necessary to evaluate the $i$-th ellipse both Linear $\mathcal{A}_{f i}^{l i n}$ and Non Linear area $\mathcal{A}_{f i}^{\text {nlin }}$.

Fig. 12. Appraisal of the proposed model for the beams tested by Dias and Barros (2008) and by Dias et al. (2007).

Fig. 13. Craking scenario regarding beam 2S-7LI45-II: numerical result for $k=1$ (a), $k=2$ (b), $k=3$ (c), and experimental post-test pictures (d-f).

Fig. 14. Comparison between numerical and experimental results: as function of the spacing between adjacent strips at $60^{\circ}$ for concrete $f_{c m} 31.1 \mathrm{MPa}$ (a) and $f_{c m} 18.6 \mathrm{MPa}$ (b); group effect for the $3^{\text {rd }}$ configuration (concrete $f_{c m} 31.1 \mathrm{MPa}$ and $\beta 60^{\circ}$ ) (c) and ideal shear strength contribution for a system of NSM with spacing $75 \mathrm{~mm}(\mathrm{~d})$. 


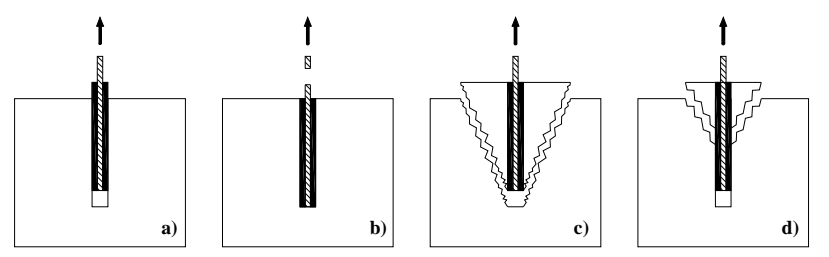

Fig. 1. Possible failure modes of an NSM FRP strip: (a) debonding, (b) laminate tensile rupture, (c) concrete semi-conical fracture, (d) mixed shallow semi-cone plus debonding.

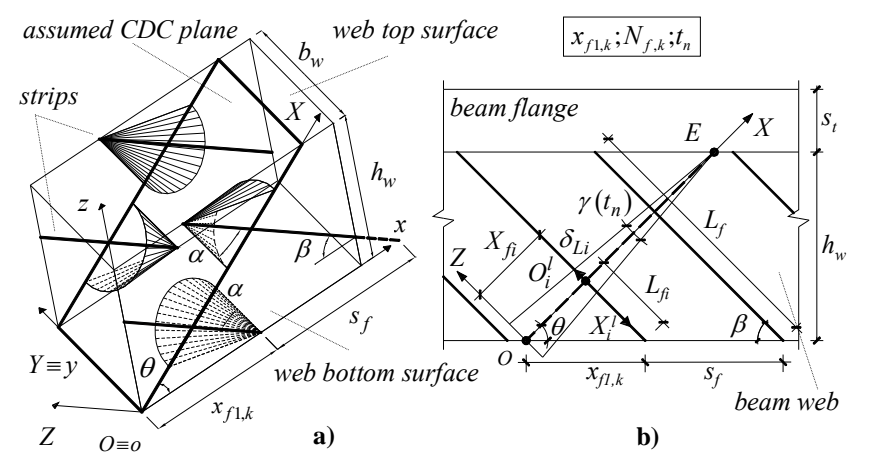

Fig. 2. RC beam web: a) axonometric view of the adopted schematization and b) shear loading process.

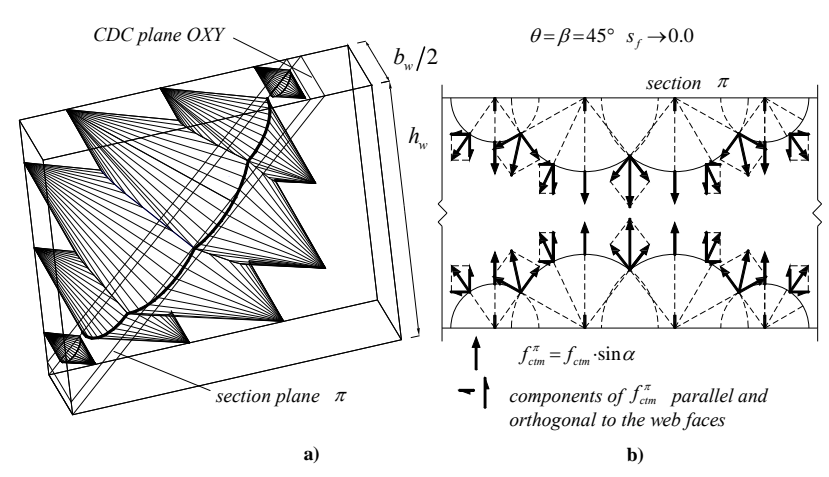

Fig. 3. Interaction among adjacent strips: a) axonometric view and b) section parallel to the CDC plane. 


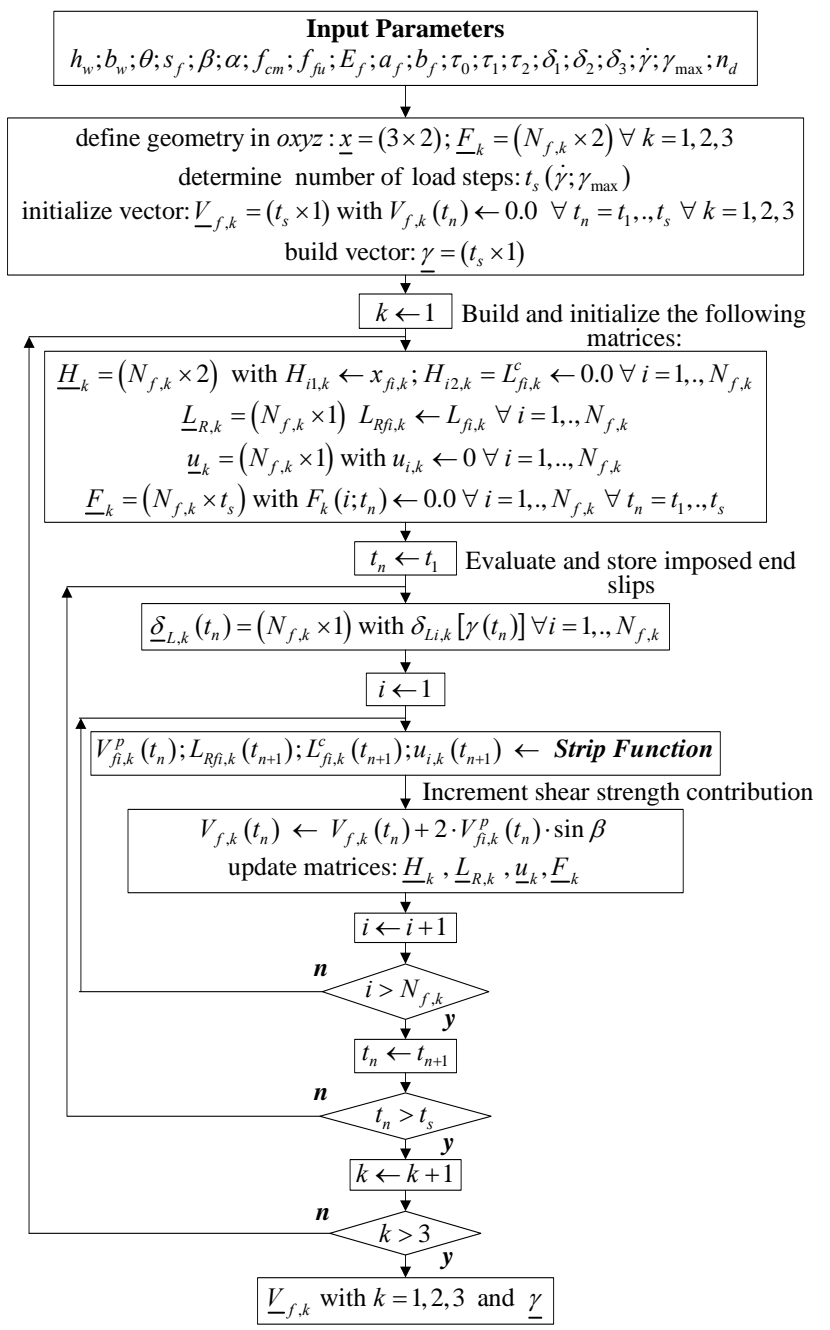

Fig. 4. Main algorithm: flow chart. 


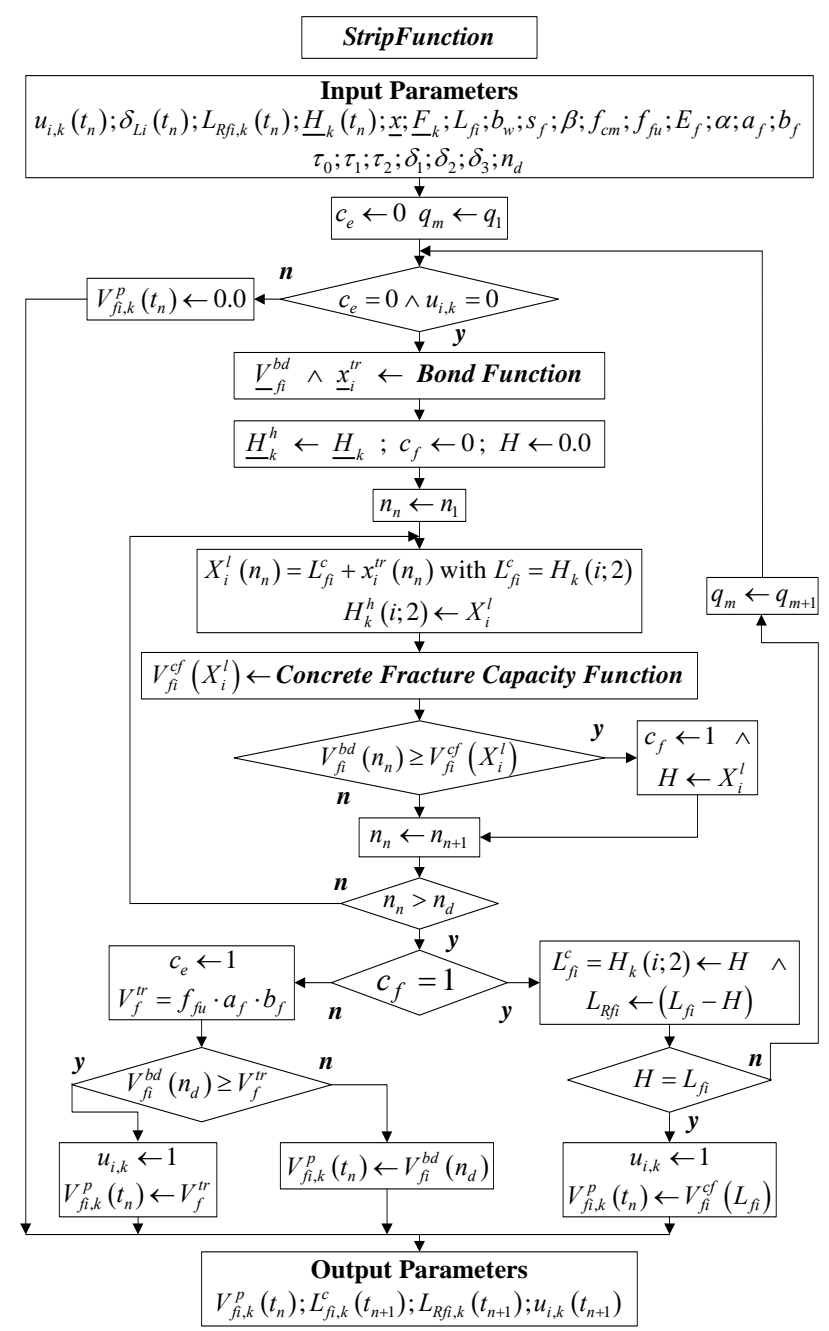

Fig. 5. Strip Function: flow chart. 

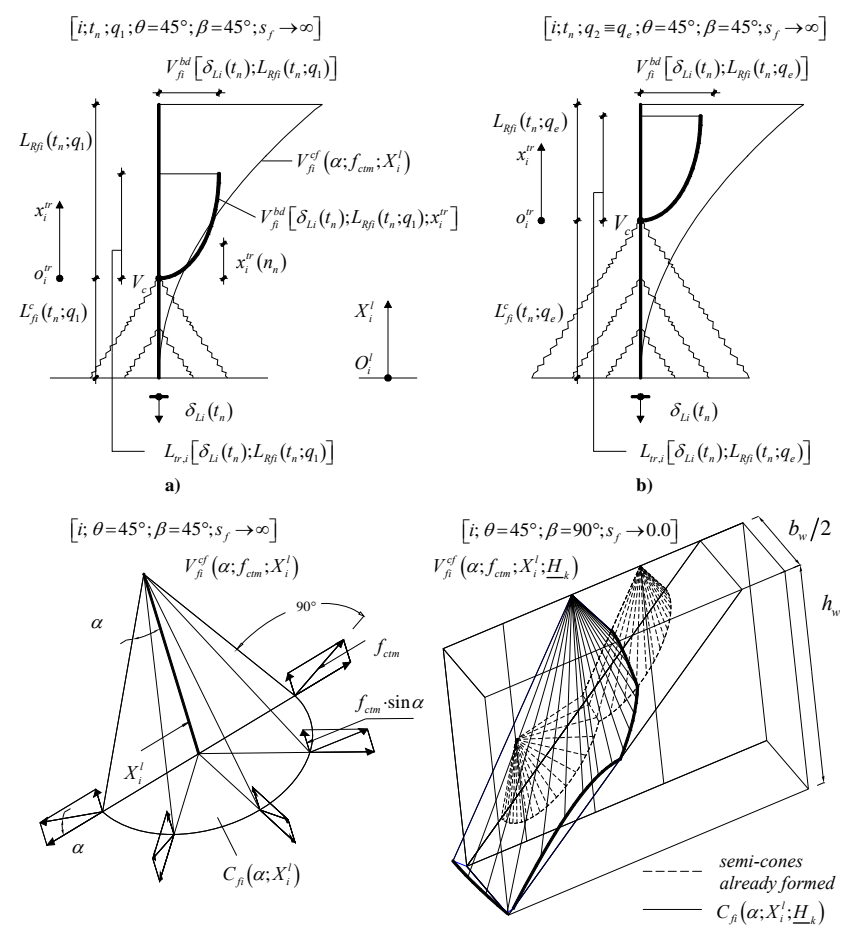

c)

d)

Fig. 6. Strip Function: (a-b) iterative procedure for searching the equilibrium condition in the surrounding concrete; (c) evaluation of the progressive concrete fracture capacity for a single slip in a simplified case and (d) in presence of interaction between adjacent strips not orthogonal to the CDC plane. 


\begin{tabular}{c} 
BondFunction \\
Input Parameters \\
$n_{d} ; f_{c m} ; L_{R f} ; \delta_{L i}\left(t_{n}\right) ; \tau_{0} ; \tau_{1} ; \tau_{2} ; \delta_{1} ; \delta_{2} ; \delta_{3} ; E_{f} ; s_{f} ; a_{f} ; b_{f} ; b_{w}$ \\
\hline
\end{tabular}

Definition of the phase undergone by the loaded end

\begin{tabular}{|c|c|c|c|}
\hline$\sqrt{ }$ & & $\checkmark$ & \\
\hline $0.0<\delta_{L i} \leq \delta_{1}$ & $\delta_{1}<\delta_{L i} \leq \delta_{2}$ & $\delta_{2}<\delta_{L i} \leq \delta_{3}$ & $\delta_{L i}>\delta_{3}$ \\
\hline
\end{tabular}

Solution of Bond for an infinite Resisting Bond Length

\begin{tabular}{|c|c|c|c|}
\hline $\begin{array}{c}L_{t r}\left(\delta_{L i}\right)=L_{t r}^{e}\left(\delta_{L i}\right) \\
C_{1}^{e} ; C_{2}^{e}\left(L_{t r}^{e}\right) ; \tau^{e}\left(x^{e}\right)\end{array}$ & \multicolumn{3}{|c|}{$\begin{array}{c}L_{t r 1}=L_{t r}^{e}\left(\delta_{1}\right) \\
C_{1}^{e} ; C_{2}^{e}\left(L_{t r 1}\right) ; \tau^{e}\left(x^{e}\right)\end{array}$} \\
\hline & $\begin{array}{c}L_{t r}\left(\delta_{L i}\right)=L_{t r 1}+L_{t r}^{s}\left(\delta_{L i}\right) \\
\quad C_{1}^{s}\left(L_{t r}^{s}\right) ; C_{2}^{s} ; \tau^{s}\left(x^{s}\right)\end{array}$ & $\begin{array}{c}L_{t r 2} \\
C_{1}^{s}\left(L_{t r 2}\right)\end{array}$ & $\begin{array}{l}=L_{t r}^{s}\left(\delta_{2}\right) \\
; C_{2}^{s} ; \tau^{s}\left(x^{s}\right)\end{array}$ \\
\hline & $\begin{array}{c}L_{t r}\left(\delta_{L i}\right)=\sum_{p=1}^{2} \\
C_{1}^{s f}\left(L_{t r}^{s f}\right) ; C\end{array}$ & $\begin{array}{l}L_{t r}^{s f}\left(\delta_{L i}\right) \\
f\left(x^{s f}\right)\end{array}$ & $\begin{array}{l}L_{t r 3}=L_{t r}^{s f}\left(\delta_{3}\right) ; C_{2}^{s f} \\
C_{1}^{s f}\left(L_{t r 3}\right) ; \tau^{s f}\left(x^{s f}\right)\end{array}$ \\
\hline & & $\begin{array}{r}L_{t r}\left(\delta_{L i}\right) \\
\quad C_{1}^{f s}(\end{array}$ & $\begin{array}{l}=\sum_{p=1}^{3} L_{t r p}+L_{t r}^{f s}\left(\delta_{L i}\right) \\
\left.L_{t r}^{f s}\right) ; C_{2}^{f s} ; \tau^{f s}\left(x^{f s}\right)\end{array}$ \\
\hline
\end{tabular}

Determination of the actual value of the Transfer Length and discretization

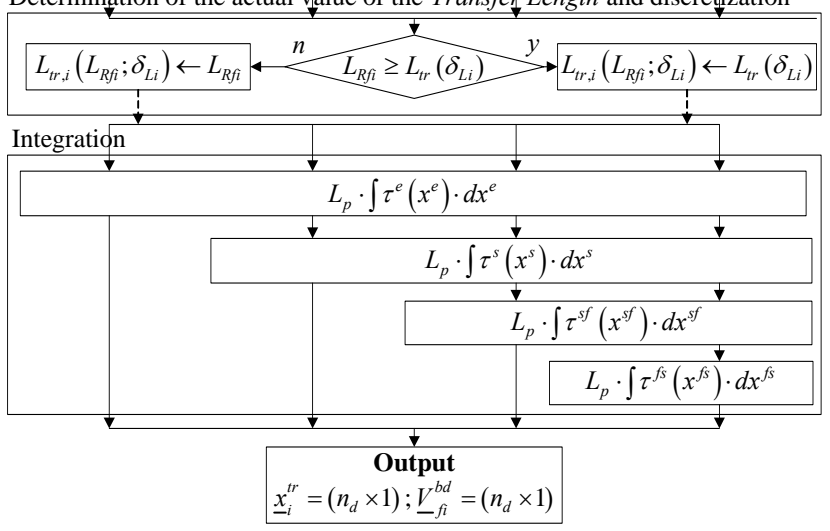

Fig. 7. Flow chart of the Bond Function. 


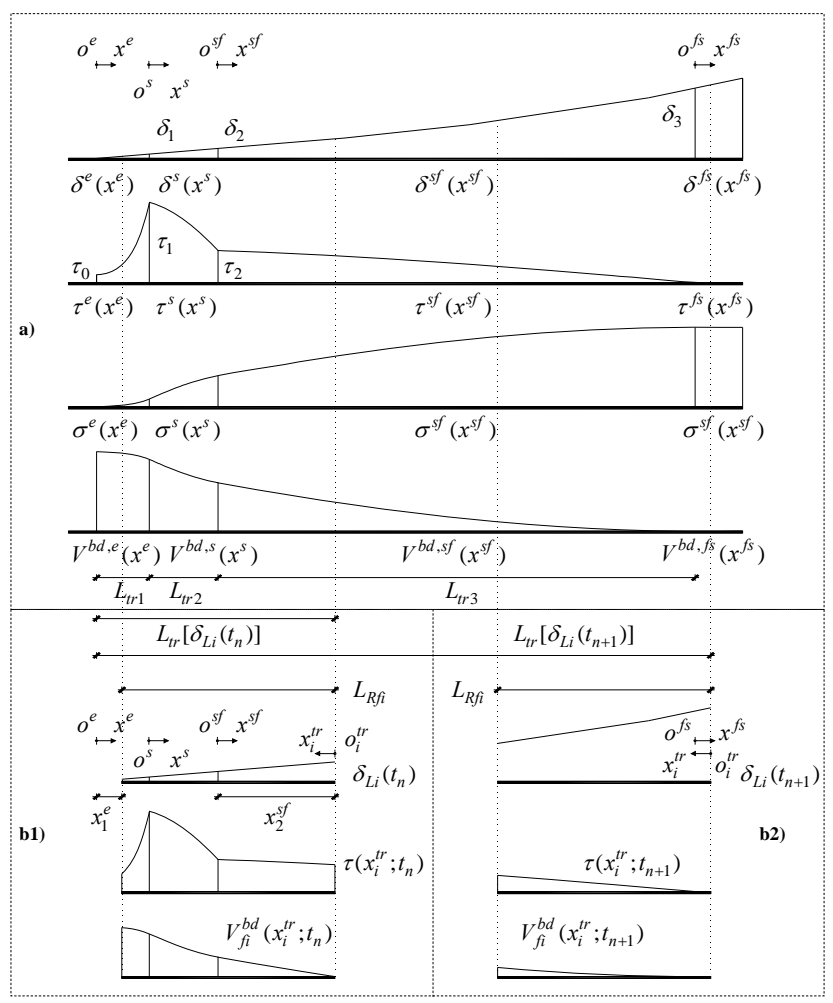

Fig. 8. Invariant bond wave: (a) evaluated for an infinite bond length; progressing from the loaded end to the free extremity for successive values of the imposed end slip $\delta_{L i}\left(t_{n}\right)$ (b1) and $\delta_{L i}\left(t_{n+1}\right)$ (b2).

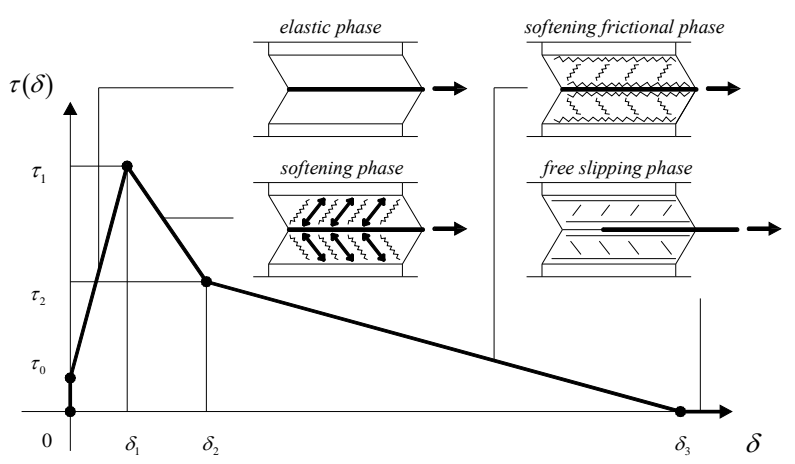

Fig. 9. Assumed local bond stress-slip relationship. 


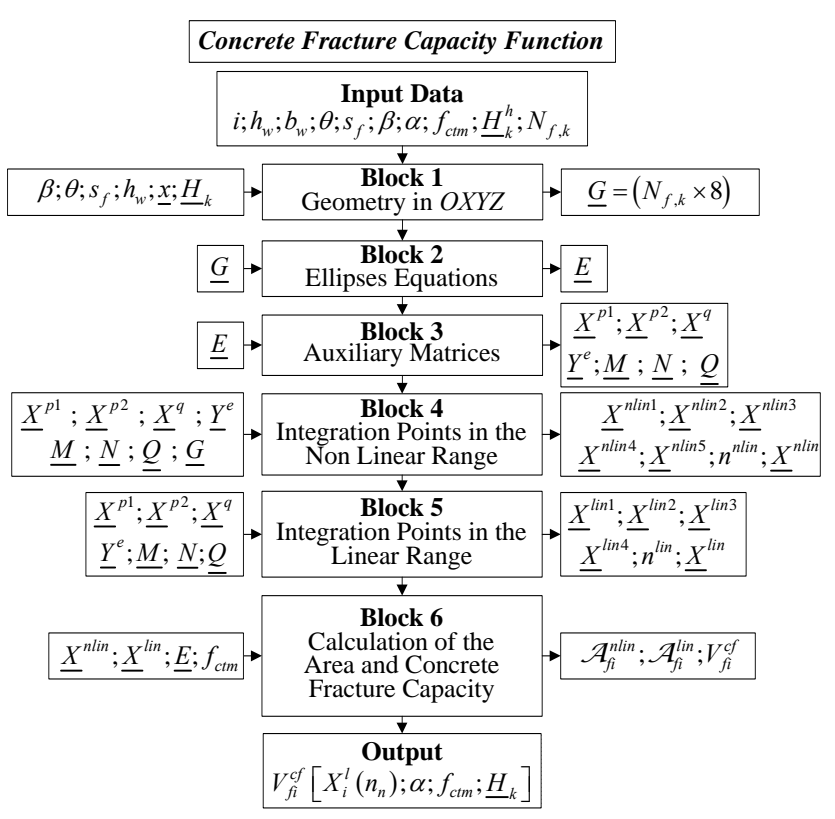

Fig. 10. Concrete Fracture Capacity Function: flow chart.

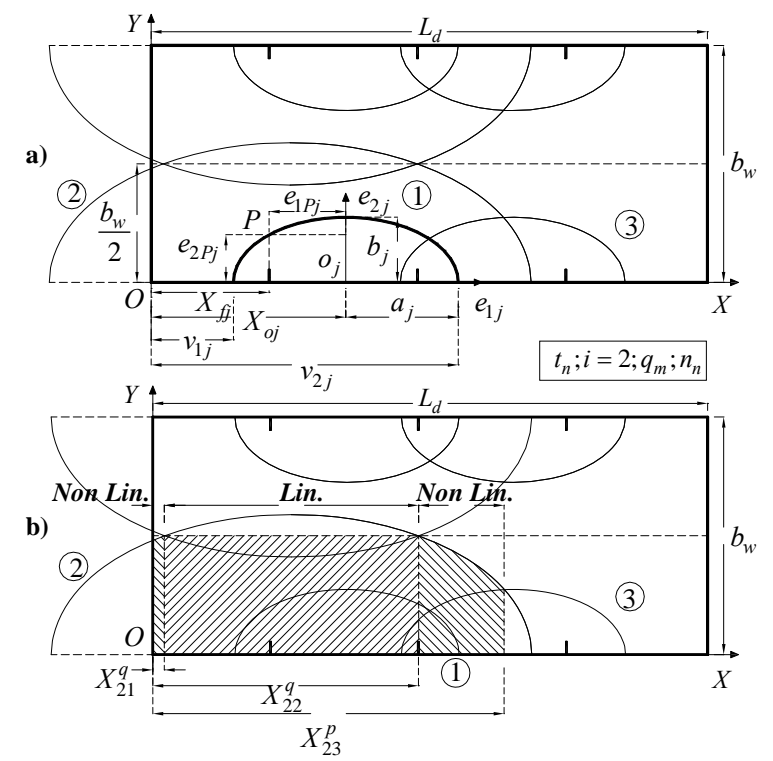

Fig. 11. CDC plane: (a) geometrical quantities in $O X Y$ and the ellipse local reference system $o_{j} e_{1 j} e_{2 j}$; (b) abscissa values necessary to evaluate the $i$-th ellipse both Linear $\mathcal{A}_{f i}^{\text {lin }}$ and Non Linear area $\mathcal{A}_{f i}^{\text {nlin }}$. 

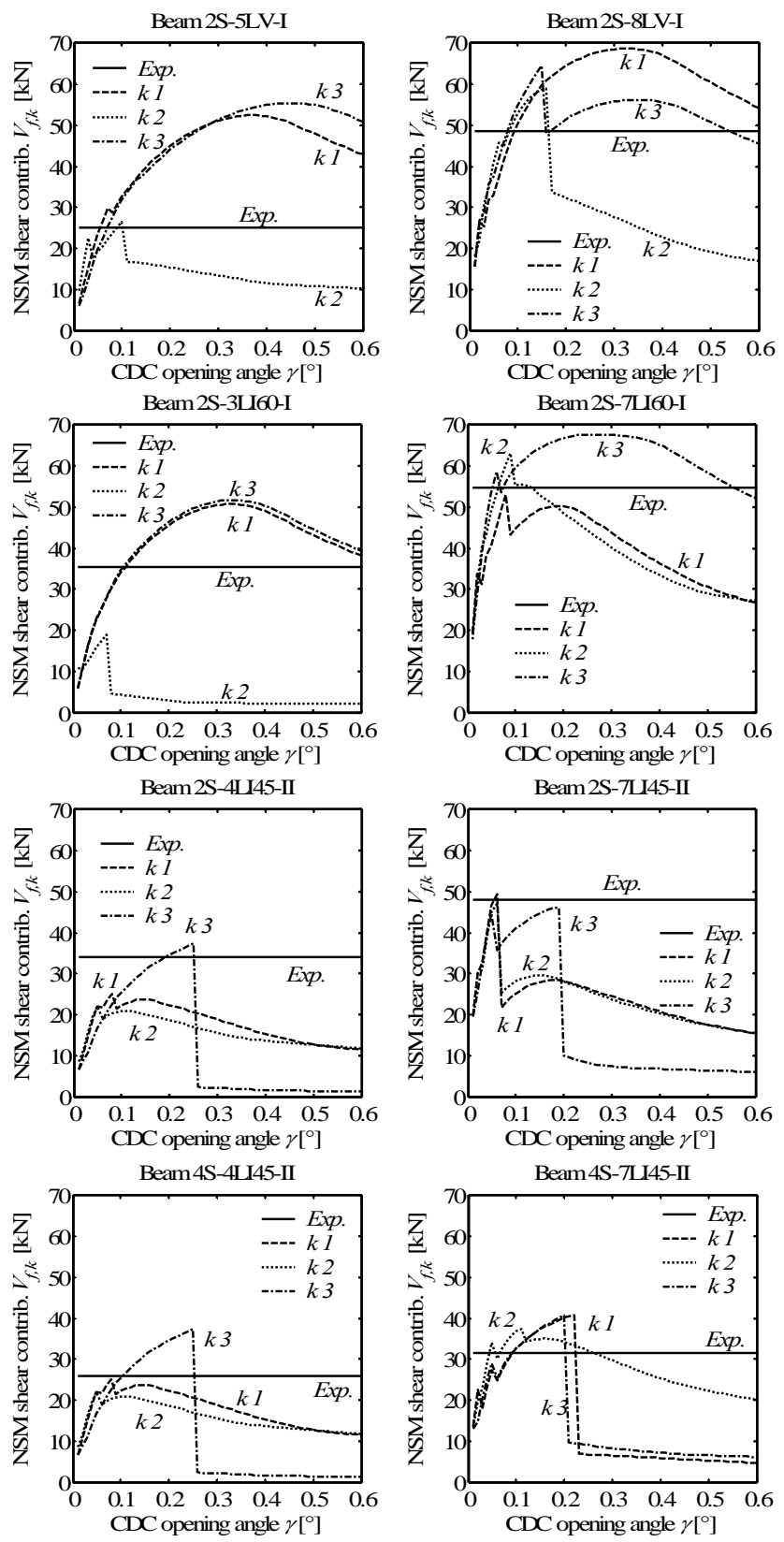

Fig. 12. Appraisal of the proposed model for the beams tested by Dias and Barros (2008) and by Dias et al. (2007). 

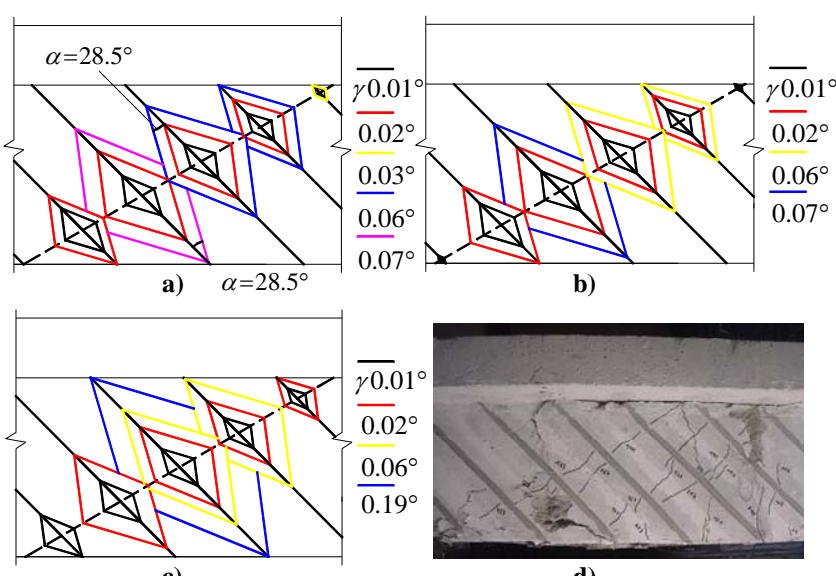

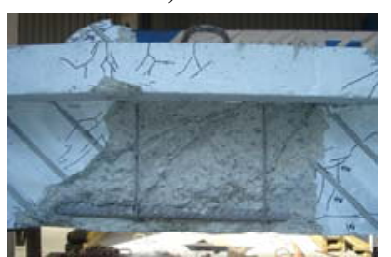

e)

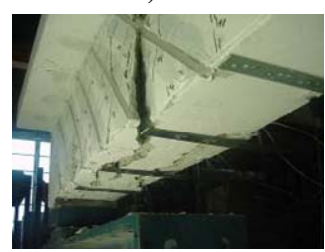

f )

Fig. 13. Craking scenario regarding beam 2S-7LI45-II: numerical result for $k=1$ (a), $k=2$ (b), $k=3$ (c), and experimental post-test pictures (d-f).

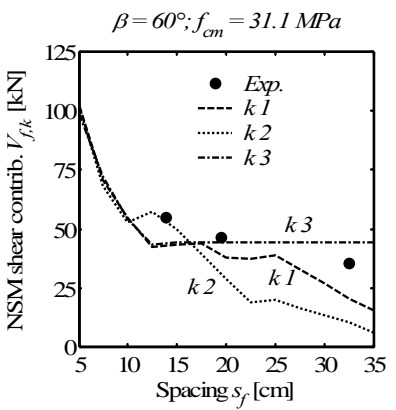

a)

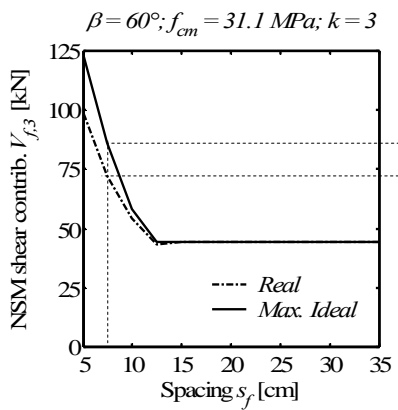

c)

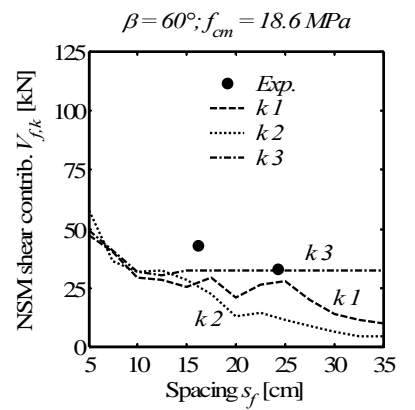

b)

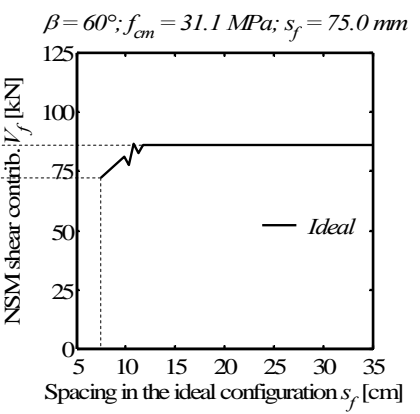

d)

Fig. 14. Comparison between numerical and experimental results: as function of the spacing between adjacent strips at $60^{\circ}$ for concrete $f_{c m} 31.1 \mathrm{MPa}$ (a) and $f_{c m} 18.6 \mathrm{MPa}$ (b); group effect for the $3^{\text {rd }}$ configuration (concrete $f_{c m} 31.1 \mathrm{MPa}$ and $\beta 60^{\circ}$ ) (c) and ideal shear strength contribution for a system of NSM with spacing $75 \mathrm{~mm}$ (d). 